\title{
Development of a Multiplex Assay for Genus- and Species-Specific Detection of Phytophthora Based on Differences in Mitochondrial Gene Order
}

\author{
Guillaume J. Bilodeau, Frank N. Martin, Michael D. Coffey, and Cheryl L. Blomquist
}

First and second authors: United States Department of Agriculture-Agricultural Research Service, Salinas, CA; third author: Department of Plant Pathology and Microbiology, University of California-Riverside; and fourth author: California Department of Food and Agriculture, Plant Pest Diagnostics Branch, Sacramento.

Current address of G. L. Bilodeau: Canadian Food Inspection Agency, Ottawa, Canada.

Accepted for publication 28 January 2014.

\begin{abstract}
Bilodeau, G. J., Martin, F. N., Coffey, M. D., and Blomquist, C. L. 2014. Development of a multiplex assay for genus- and species-specific detection of Phytophthora based on differences in mitochondrial gene order. Phytopathology 104:733-748.

A molecular diagnostic assay for Phytophthora spp. that is specific, sensitive, has both genus- and species-specific detection capabilities multiplexed, and can be used to systematically develop markers for detection of a wide range of species would facilitate research and regulatory efforts. To address this need, a marker system was developed based on the high copy sequences of the mitochondrial DNA utilizing gene orders that were highly conserved in the genus Phytophthora but different in the related genus Pythium and plants to reduce the importance of highly controlled annealing temperatures for specificity. An amplification primer pair designed from conserved regions of the atp 9 and nad 9 genes produced an amplicon of $\approx 340 \mathrm{bp}$ specific for the Phytophthora spp. tested. The TaqMan probe for the genus-specific Phytophthora test was designed from a conserved portion of the atp 9 gene whereas variable intergenic spacer sequences were used for designing the species-

species-specific probes could be developed for at least 70 additional described and provisional species; the use of locked nucleic acids in TaqMan probes should expand this list. A second locus spanning three tRNAs (trnM-trnP-trnM) was also evaluated for genus-specific detection capabilities. At $206 \mathrm{bp}$, it was not as useful for systematic development of a broad range of species-specific probes as the larger 340-bp amplicon. All markers were validated against a test panel that included 87 Phytophthora spp., 14 provisional Phytophthora spp., 29 Pythium spp., 1 Phytopythium sp., and 39 plant species. Species-specific probes were validated further against a range of geographically diverse isolates to ensure uniformity of detection at an intraspecific level, as well as with other species having high levels of sequence similarity to ensure specificity. Both diagnostic assays were also validated against 130 environmental samples from a range of hosts. The only limitation observed was that primers for the $340 \mathrm{bp}$ atp9-nad9 locus did not amplify Phytophthora bisheria or $P$. frigida. The identification of species present in a sample can be determined without the need for culturing by sequencing the genus-specific amplicon and comparing that with a reference sequence database of known Phytophthora spp.
\end{abstract} specific TaqMan probes. Specific probes were developed for 13 species and the P. citricola species complex. In silico analysis suggests that
Additional keywords: real-time PCR.
Species in the genus Phytophthora have had a significant impact on production of economic crop plants and the health of native plant communities. The importance of members of the genus as plant pathogens infecting a wide range of host species is shown by the $>11,500$ entries in the Systematic Mycology and Microbiology Lab website listing fungal-host reports (http://nt. ars-grin.gov/fungaldatabases/fungushost/FungusHost.cfm). This number is likely to increase in the future, as the number of newly discovered and described species continues to increase. From 2000 to 2007, the number of described species in this genus essentially doubled (9) and, whereas the recent taxonomic updates of the genus by Kroon et al. (27) lists 105 described species, a later review noted 117 species (32). However, these listings are already out of date because there have been a number of new

Corresponding author: F. N. Martin; E-mail address: frank.martin@ars.usda.gov

* The $\boldsymbol{e}$-Xtra logo stands for "electronic extra" and indicates that the online version contains three supplementary tables and sequence alignments of the two loci used for marker design.

http://dx.doi.org/10.1094/PHYTO-09-13-0263-R

This article is in the public domain and not copyrightable. It may be freely reprinted with customary crediting of the source. The American Phytopathological Society, 2014. species described as well as provisional species named since these manuscripts were published.

Although the increased availability of sequence data for confirming species identification and conducting phylogenetic analysis has contributed to this increase in species description, perhaps equally important is the increased number of field surveys in forest and commercial nursery environments that have resulted in the identification of new species. An example of this in the United States can be seen with the quarantine species Phytophthora ramorum, which was initially found in the forest ecosystem of central coastal California but has since spread to other parts of coastal California and southern Oregon $(16,38)$. P. ramorum has been found in the nursery industry and been transported on infected nursery stock from west coast to east coast as well as between states within the eastern and western United States $(15,18)$. Significant effort has been put into surveys to determine the extent of pathogen spread and the inspection of plant shipments to prevent introductions into new locations. This has provided a much better picture of other Phytophthora spp. present in the various ecosystems investigated and provided isolates of previously undescribed species. Surveys for $P$. ramorum, $P$. kernoviae, and $P$. alni, which have caused significant disruptions to natural ecosystem in Europe (9-11), have also resulted 
in the isolation and description of previously unknown species in Europe.

Given the role of international trade in moving plant material around the world, the consequence of the introduction of invasive species on native ecosystems, and the regulatory needs surrounding the plant trade industry to prevent movement of quarantine species, having a robust molecular diagnostic capability for detection of Phytophthora spp. is essential. An ideal marker system would be highly sensitive, so that the pathogen could be detected when present in low amounts; be capable of detecting the pathogen at both a species- and genus-specific level; and allow for the systematic development of species-specific markers. Of particular importance is the availability of a robust genus-specific detection capability that can be run concurrently with species-specific detection. This would allow for a broader analysis of any species that may be present in a sample or ecosystem rather than just be able to determine whether an individual species is present. With the availability of a sequence database of the target locus for the genus, it would be possible to characterize the species community without the need for culturing.

A variety of markers have been designed for conventional and real-time polymerase chain reaction (PCR) assays for Phytophthora spp. (32). Although the design of some of these has focused on a particular species using a unique random clone, an amplicon from a random amplified polymorphic DNA assay, or a unique sequence differences in a specific gene, several loci that are conserved within a species but variable between them have been used for the systematic development of multiple species-specific diagnostic markers for conventional and real-time PCRs. The internal transcribed spacer (ITS) region of the ribosomal DNA (rDNA) has been commonly used for this purpose, as have introns of the Ras-related Yptl gene and the spacer region between the coxl and cox 2 mitochondrially encoded genes (32). Although a genus-specific detection capability for real-time PCR has been reported for the ITS-based diagnostic assay for P. ramorum (26) it is not fully genus specific because several Pythium spp. were also detected. Likewise, although there is a genus-specific detection capability using the spacer region between the coxl and cox 2 genes, this is dependent on control of the annealing temperature for specificity and is not suitable for real-time PCR diagnostic techniques (34). A genus-specific detection capability has been described for the Ras-related Yptl gene but this is with conventional PCR and the $\approx 470$-bp amplicon may not be suitable for real-time PCR $(40,41)$. A real-time PCR probe for Phytophthora spp. has been developed by Bilodeau et al. (5) using the $\beta$-tubulin region but has not been fully validated against a range of Pythium spp.

When developing molecular diagnostic techniques for pathogen detection, it is important to keep in mind that the assay must be reproducible and accurate as well as capable of high-throughput sample processing, with different operators using different equipment in different locations. For example, a variety of thermal cyclers may be used in different facilities and, if a narrow window for annealing temperature is necessary for accurate results, then additional revalidation of thermocyclers to account for variation in temperature calibration and ramping speed among machines may be required. Amplification conditions may also need to be monitored consistently as a means for ensuring the accuracy of results. This requirement increases the amount of work needed to complete the assays but also the potential for unnecessary falsepositive and false-negative results and problems with reproducibility of results between different diagnostic facilities. The capability of high-throughput sample processing is also required for some diagnostic labs; therefore, the ability to obtain accurate results with an assay using either a 96-well plate or single tubes is essential.

In an effort to improve the diagnostic capability for Phytophthora spp., this study approached the design of a new marker sys- tem with several goals in mind: (i) a desire to limit the importance of precisely controlled annealing temperature for maintaining specificity by selecting primer annealing sites that were adjacent in Phytophthora spp. but separated in Pythium spp. and plants, (ii) a robust genus-specific detection capability was needed that could be multiplexed with the species-specific marker, (iii) a desire to identify a locus that would allow for the systematic development of species-specific markers for a wide range of species, (iv) the target sequence needed to be a high copy number to provide the greatest sensitivity of detection, and (v) the diagnostic marker system needed to include a plant amplicon to serve as a positive control. Due to its high copy number, the mitochondrial genome was selected for designing this new marker system. Having the sequenced mitochondrial genomes for 20 Phytophthora and 14 Pythium spp. (F. Martin, unpublished data) provided the opportunity to identify gene orders that were highly conserved in the genus Phytophthora but different in the genus Pythium and plants and that could be used for the design of genus-specific amplification primers. Rather than have an amplicon used for genusspecific detection and a second amplicon for species-specific detection, the genus-specific primers were designed so that the amplicon included a highly conserved region that could be used for annealing a genus-specific TaqMan probe as well as variable intergenic spacer sequences for design of species-specific TaqMan probes. Sequencing these regions from a wide range of species allowed us to evaluate the feasibility of this location for the design of species-specific TaqMan probes. This report describes the design of two marker systems and validation of their specificity with culture and environmental samples.

\section{MATERIALS AND METHODS}

Phytophthora, Pythium, Phytopythium, and plant species used. The 667 isolates used in this investigation represented 87 valid and 14 provisional Phytophthora spp. (95 of these were used for the core plate used to evaluate all primers and probes) and were from the World Phytophthora Genetic Resource Collection at the University of California, Riverside (Table 1). Cultures were grown and DNA extracted as previously described (8). Some additional isolates of $P$. megakarya and $P$. palmivora recovered from cacao in Ghana were also included in the analysis (DNA samples kindly provided by F. Govers). Twenty-nine Pythium spp. were used to confirm the specificity of the assay (Table 2); information on their culture and DNA extraction can be found in Martin (30). A range of plant species were also used to evaluate the specificity (Table 2). DNAs were extracted as previously noted (34) or were part of the environmental sample evaluation and were extracted using the United States Department of Agriculture-Animal and Plant Health Inspection Service (USDAAPHIS)-approved DNA extraction protocol (46). To verify the presence of amplifiable Pythium DNA, real-time PCR with SYBR Green was performed using universal primers ITS1 and ITS4 (47). PCR was performed using SYBR Green PCR Master Mix 1× (Applied Biosystems, Carlsbad, CA) primers ITS1 and ITS4 at $0.4 \mu \mathrm{M}$. PCR analysis was performed in a reaction volume of $25 \mu \mathrm{l}$ on the ICycler instrument (IQ4; Bio-Rad Laboratories, Hercules, CA). Amplification conditions were one cycle of activation at $95^{\circ} \mathrm{C}$ for $8.5 \mathrm{~min}$; followed by 40 cycles of $95^{\circ} \mathrm{C}$ for $30 \mathrm{~s}, 60^{\circ} \mathrm{C}$ for $30 \mathrm{~s}$, and $72^{\circ} \mathrm{C}$ for $60 \mathrm{~s}$; finishing with a final cycle of $72^{\circ} \mathrm{C}$ for $10 \mathrm{~min}$. Melt-curve analysis started at $60^{\circ} \mathrm{C}$ and increased $0.5^{\circ} \mathrm{C}$ every two cycles 70 times, with a final incubation at $4^{\circ} \mathrm{C}$. To confirm the suitability of plant DNA for PCR amplification, the TaqMan internal plant control probe and primers were used, as described below.

Identification of mitochondrial DNA regions for marker development. Mitochondrial genome data for $P$. infestans, $P$. ramorum (33), P. sojae, and 17 other Phytophthora spp. and 14 Pythium spp. are part of an ongoing mitochondrial genome 
sequencing project (F. Martin, unpublished data) which provided us the opportunity to use comparative genomic approaches to identify regions of the genome that might be useful for developing diagnostic markers for Phytophthora spp. Plant mitochondrial sequences used for this studies were obtained from GenBank. Gene order differences that were conserved among Phytophthora spp. but different in Pythium spp. and plants were identified. Three regions were the focus of this investigation: trnM-trnP-trnM, atp9-nad9, and nad11-secY. In Pythium spp., the gene order trnM-trnP was conserved but the last trnM was located $>10 \mathrm{~kb}$ away and in the opposite orientation, atp 9 and nad 9 were located 18 to $30 \mathrm{~kb}$ apart, and nadll and $\sec Y$ were at least $15 \mathrm{~kb}$ apart. This may be observed using comparative genomics of mitochondrial genomes deposited in GenBank (AY894827, AY894828, AY894835, DQ832717, DQ832718, and NC002387 for Phytophthora spp. and NC014280 for Pythium ultimum).

Amplification and sequencing. For sequencing of the trnMtrnP-trnM locus, amplification primers were selected from the flanking genes (lrn and rpll4); amplification primers for the two other loci were in the indicated genes (Table 3). All amplifications were performed using $\approx 10 \mathrm{ng}$ of template DNA, $0.4 \mathrm{mM}$ forward and reverse primers, $2 \mathrm{mM} \mathrm{MgCl}_{2}, 200 \mu \mathrm{M}$ dNTP, $1 \times$ amplification buffer, and 1 unit of AmpliTaq (Applied Biosystems) in a volume of $25 \mu \mathrm{l}$. Templates were amplified in an ABI 9600 thermal cycler with the following cycling conditions: (i) for TrnM-TrnM, one interval of $95^{\circ} \mathrm{C}$ for $3 \mathrm{~min} ; 35$ cycles of $95^{\circ} \mathrm{C}$ for $1 \mathrm{~min}, 1 \mathrm{~min}$ of annealing at $61^{\circ} \mathrm{C}$, and extension at $72^{\circ} \mathrm{C}$ for $2 \mathrm{~min}$; and one interval of $72^{\circ} \mathrm{C}$ for $5 \mathrm{~min}$ followed by a $4^{\circ} \mathrm{C}$ hold; (ii) for atp9-nad9, one interval of $95^{\circ} \mathrm{C}$ for $3 \mathrm{~min}$; 40 cycles of $95^{\circ} \mathrm{C}$ for $1 \mathrm{~min}, 1 \mathrm{~min}$ of annealing at $61^{\circ} \mathrm{C}$, and extension at $72^{\circ} \mathrm{C}$ for $1 \mathrm{~min}$; and one interval of $72^{\circ} \mathrm{C}$ for $10 \mathrm{~min}$ followed by a $4^{\circ} \mathrm{C}$ hold; and (iii) for nad11-secY, 1 interval of $95^{\circ} \mathrm{C}$ for $3 \mathrm{~min}$; 40 cycles of $95^{\circ} \mathrm{C}$ for $30 \mathrm{~s}, 45 \mathrm{~s}$ of annealing at $51^{\circ} \mathrm{C}$, and extension at $72^{\circ} \mathrm{C}$ for $1 \mathrm{~min}$; and one interval of $72^{\circ} \mathrm{C}$ for $5 \mathrm{~min}$ followed by a $4^{\circ} \mathrm{C}$ hold. After confirming template amplification by running samples on an agarose gel, sequencing templates were treated with ExoSap (USB, Cleveland) according to the manufacturer's instructions and sent to the Penn State Genomics Core Facility of the Huck Institute for Life Sciences (University Park, PA) for sequencing with the amplification primers. Each template was sequenced in both directions to generate a consensus sequence based on complementary strands. Sequencher 4.7 (Gene Codes, Ann Arbor, MI) was used to generate consensus sequences while DS Gene v. 2.5 (Accelrys, San Diego, CA) was used for making alignments.

Marker development. Sequence alignments were examined for highly conserved regions for design of amplification primers and a genus-specific TaqMan probe. Intergenic spacer sequences were also examined for GC content and whether the level of sequence divergence was appropriate for development of speciesspecific TaqMan probes. The IDT SciTools OligoAnalyser 3.1 (Integrated DNA Technologies Inc., Coralville, IA) was used for primer design. Specific primers were designed so that the nucleotides unique to the target were at the $3^{\prime}$ end position of the primer and a TaqMan probe (20) could be designed in the middle of the amplicon when possible. For TaqMan probe design, the following parameters (6) were used, with some exceptions because of the high A-T content of the these mitochondrial region: melting temperature $\left(\mathrm{T}_{\mathrm{m}}\right)$ of the TaqMan probe was selected to be $10^{\circ} \mathrm{C}$ higher (when possible; if not, then at least 3 to $5^{\circ} \mathrm{C}$ degree higher than the amplification primers) and 15 to $36 \mathrm{bp}$ in length, with the total number of Gs or Cs in the last five nucleotides at the $3^{\prime}$ end of the probe not exceeding two. Mismatching nucleotides responsible for species specificity were positioned as close as possible to the middle of the probe rather than at the ends while avoiding positions with secondary structure (14). The $5^{\prime}$ end of the atp9-nad9 TaqMan probe for the genus Phytophthora was labeled with FAM (fluorescein), and other probes tested with
CAL Fluor Red, Hex, or Cy5 (Table 3). The trnM-trnM Phytophthora genus-specific probe was labeled with Hex. The $3^{\prime}$ end of all probes was labeled with the Black Hole Quencher-1 (BHQ-1; Biosearch Technologies, Inc., Novato, CA) or BHQ-2, depending on the TaqMan assay. The amplification master mix contained the genus-specific primer pair at $0.5 \mu \mathrm{M}$ (some primers had a $5^{\prime}$ flap to improve the real-time PCR signal) (1). Depending on the assay, the TaqMan probe concentration varied; it was $0.05 \mu \mathrm{M}$ for atp9nad9 Phytophthora genus-specific, Phytophthora cactorum, $P$. cambivora, $P$. fragariae, and $P$. syringae species-specific probes; $0.1 \mu \mathrm{M}$ for trnM-trnM Phytophthora genus-specific, P. alni, $P$. cinnamomi, $P$. citricola complex, $P$. kernoviae, $P$. lacustris, $P$. nicotianae, $P$. palmivora, $P$. pseudosyringae, and $P$. rubi speciesspecific probes; or $0.025 \mu \mathrm{M}$ for the $P$. ramorum species-specific probe. Other components of the amplification mix included addition of $6 \mathrm{mM} \mathrm{MgCl} 2$ and Real Master Mix without Rox (5 Prime; Fisher Scientific Company, LLC, Waltham, MA), with PCR cycling conditions set at $95^{\circ} \mathrm{C}$ for $2 \mathrm{~min}, 50$ cycles at $95^{\circ} \mathrm{C}$ for $15 \mathrm{~s}$, and the appropriate annealing temperature for each species-specific TaqMan probe $\left(62^{\circ} \mathrm{C}\right.$ for the trnM-trnM genusspecific assay and the temperatures indicated in Table 4 for the remaining markers) for $1 \mathrm{~min} 30 \mathrm{~s}$, in a reaction volume of $25 \mu \mathrm{l}$ on the ICycler instrument. The primers for the plant internal control were present at $0.0125 \mu \mathrm{M}$ and the probe, which was labeled with CAL Fluor Red and BHQ-2, at $0.01 \mu \mathrm{M}$.

Testing of the marker systems for specificity and sensitivity. Two loci, the trnM-trnM and atp9-nad9 regions (Fig. 1), were tested for genus specificity on a core of 87 described and 14 provisional Phytophthora spp. (101 total) (Table 1), 29 Pythium spp. (Table 2), and 39 plant species (Table 2). The intergenic spacer regions of the atp9-nad9 locus was chosen for design of the species-specific TaqMan probes because this region contained a higher level of sequence divergence than the trnM-trnM locus. Fourteen species-specific TaqMan probes (Table 3) were tested with the core Phytophthora sp. plate (Table 1) for specificity. Probes were tested on multiple isolates of the species for which they were designed and other species that had similar sequences at probe annealing sites. The $P$. ramorum and $P$. kernoviae speciesspecific probes were tested on a panel of additional species with 256 isolates of Phytophthora (94 species, with 1 to 6 isolates/ species). To determine the limit of sensitivity for genus- and species-specific detection, standard curves using a serial dilution from $1 \mathrm{ng}$ to $10 \mathrm{fg}$ of stock DNA of the target species were evaluated.

Evaluation on field samples. Fields samples were received as a blind test for evaluation with the two Phytophthora genusspecific TaqMan assays and the atp9-nad9 species-specific assay. All samples were run in multiplex with the plant internal control to confirm the ability to amplify the DNA sample.

California Department of Food and Agriculture field samples. Ninety-nine plant samples submitted to the California Department of Food and Agriculture (CDFA) from different locations in Californian had DNA extracted using the standard USDA-APHIS procedure for $P$. ramorum (46). All samples were tested with both the atp9-nad9 and trnM-trnM Phytophthora genus-specific markers. The atp-9-nad9 assay was multiplexed with P. ramorum and $P$. kernoviae species-specific probes, with the following speciesspecific probes tested individually: $P$. syringae, $P$. cambivora, $P$. citricola group, and $P$. pseudosyringae. To confirm the species that were present, the genus-specific amplicon was sequenced for all samples that were positive for a Phytophthora $\mathrm{sp}$. and the sequence compared with our sequence database by BLAST analysis using BioEdit ver. 7.0.9.0 (19).

Big Sur. Field samples were collected on 2 June 2010 at Pfeiffer Big Sur State Park and Andrew Molera State Park, Monterey County, CA. Leaf pieces with lesions and nonsymptomatic leaves were cut with a number 3 cork borer, with half of the leaf disk plated on pimaricin-ampicillin-rifampicin-pentachloronitro- 
TABLE 1. Phytophthora, Pythium, and Phytopythium spp. isolate numbers and origins included in this investigation

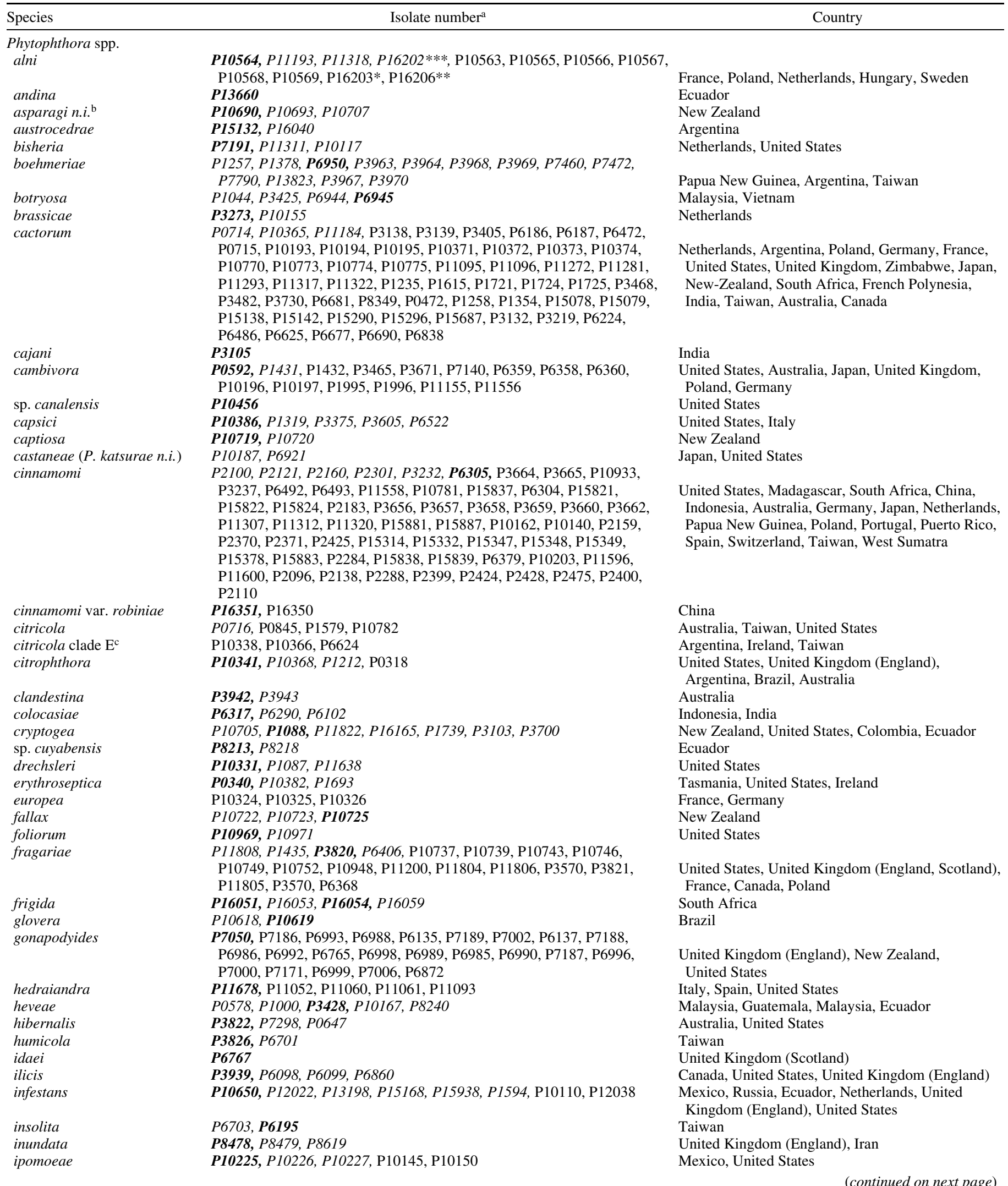

(continued on next page)

a Additional information on isolates (hosts) may be obtained at the website for the World Phytophthora Genetic Resource Collection at the University of California, Riverside (available online : http://phytophthora.ucr.edu/databasemain.html); isolates starting with the letter "P" are from this collection. Isolates of all species used for the core plate are in bold face. All isolate numbers in italic were tested with the P. ramorum and P. kernoviae, ATP9-NAD9 Phytophthora genus and TrnM Phytophthora genus probes. Asterisks: $*=$ subspecies alni (type), $* *=$ subspecies uniformis (type), and $* * *=$ subspecies multiformis (type). Isolates starting with a GH are from Ghana and DNA was provided by F. Govers.

${ }^{\mathrm{b}}$ Initials n.i. = noma invalidum; a well-characterized species but the name is not valid.

c P. citricola clade E refers to the classification of Jung and Burgess (25).

d Not determined. 
TABLE 1. (continued from preceding page)

\begin{tabular}{|c|c|c|}
\hline Species & Isolate number ${ }^{\mathrm{a}}$ & Country \\
\hline iranica & P3882 & Iran \\
\hline sp. kelmania & P10613, P1810, P10614 & United States \\
\hline kernoviae & P10681, P10956, P10958, P10957, P10671 & New Zealand, United Kingdom (England) \\
\hline lacustris & P10337, P10283, P10284 & United Kingdom \\
\hline sp. lacrimae & $P 15880$ & Netherlands \\
\hline sp. lagoariana & P8220, P8223 & Ecuador \\
\hline lateralis & P3361, P1728, P3888 & United States \\
\hline macrochlamydospora & P10267, P8017 & Australia \\
\hline meadii & P6128 & India \\
\hline medicaginis & P0127, P10683, P7029 & Australia, United States \\
\hline megakarya & $\begin{array}{l}\text { P1664, P1672, P8516, GH-AR 06, GH-AR 08, GH-AR 15, GH-AR 16, } \\
\text { GH-AR 18, GH-BAR 17, GH-BAR 21, GH-BAR 26, GH-BAR 28, } \\
\text { GH-VR 04, GH-VR 09, GH-VR 10, GH-VR 13, GH-WR 21, } \\
\text { GH-WR 47, GH-WR 51, GH-WR 56, GH-WR 60, 327, } 328\end{array}$ & Nigeria, Cameroon, Sao Tome, Ghana \\
\hline megasperma & P10340, P1679, P3136, Р3600, P6957 & Australia, Japan, United States \\
\hline melonis & P3609, P6870, P1475, P1748 & Japan, China \\
\hline mengei & P127, P1275 & United States \\
\hline mexicana & P0646 & Mexico \\
\hline mirabilis & P10231, P3005, P3010, P3007 & Mexico \\
\hline multivesiculata & $P 10670$ & New Zealand \\
\hline multivora & P7902, P10300, P10458, P10977, P11094, P11569, P11832, P1817 & South Africa, Spain, United States \\
\hline sp. napoensis & $P 8225, P 8221$, P8222 & Ecuador \\
\hline nemorosa & P10288, $P 16352$ & United States \\
\hline nicotianae & $\begin{array}{l}\text { P10297, P10381, P6915, P7146, P1325, P10381, P6915, P7146, P3458, } \\
\text { P10802, P10297, P6915, P1494, P7665, P7387, P7330, P1751, P1753, } \\
\text { P1752, P7346, P6113, P7522, P0700, P3815, P1577, P6115, P1955, } \\
\text { P7622, P16870, P16883, P1083, P1452, P16824, P3813, P1350, P1495, } \\
\text { P0583, P1333, P1334, P1335, P0582, P16823, P3234, P6832, P3549, } \\
\text { P3456, P7449, P3461, P3118, P7561 }\end{array}$ & $\begin{array}{l}\text { United States, China, Germany, Mexico, Venezuela, } \\
\text { Japan, Australia, Ponape, } \\
\text { South Africa, Spain, Greece, Pakistan, India, } \\
\text { United Kingdom, Philippines }\end{array}$ \\
\hline sp. niederhauserii & P10279, P10617, P10616, P10976, P16237, P16384, P7377 & Hungary, United States, Netherlands \\
\hline sp. novaeguineae & P3389, $P 1256$ & Papua New Guinea \\
\hline sp. ohioensis & $P 16050$ & United States \\
\hline palmivora & $\begin{array}{l}\text { P6390, P0255, P0113, P0376, P0633, P0739, P10212, P10213, P10272, } \\
\text { P10296, P10336, P10366, P10420, P10422, P10423, P10425, P10769, } \\
\text { P10817, P10818, P11005, P11007, P11009, P11010, P11011, P11012, } \\
\text { P11013, P11026, P11099, P11175, P1182, P11851, P15825, P16385, } \\
\text { P3249, P3502, P6213, P6218, P6375, P7090, P7537, P7551, P8690, } \\
\text { P8702, P8766, GH-WR 61, GH-WR 38, GH-ER 18, GH-CR 15, } \\
\text { GH-BAR 13, GH-BAR 12, GH-AR 22, 329, P10213 }\end{array}$ & $\begin{array}{l}\text { Indonesia, Costa Rica, United States, American } \\
\text { Samoa, Argentina, Ghana, Guam, Indonesia, } \\
\text { Malaysia, Philippines, Windward Island }\end{array}$ \\
\hline parsiana & $P 21282, P 21281$ & Iran, United States \\
\hline parvispora & P8495, P7154,P8494, P6378, P2404 & Germany, Israel, Taiwan \\
\hline sp. personii & $P 11555$ & United States \\
\hline sp. PgChlamyo & P10669, P6133, P6983, P6997, P6134, P6138 & New Zealand, United Kingdom, United States \\
\hline phaseoli & P6609, P10150, P10145 & United States \\
\hline pini & $\begin{array}{l}\text { P0767, P10204, P10762, P10763, P10764, P10765, P11154, P1632, } \\
\text { P1801, P1806 }\end{array}$ & Canada, Poland, United States \\
\hline pinifolia & $P 16100$ & Chile \\
\hline pistaciae & P6197, $P 6196$ & Iran \\
\hline plurivora & $\begin{array}{l}\text { P1805, P0316, P0768, P10185, P10189, P10623, P10627, P10679, } \\
\text { P11058, P11100, P11386, P11425, P11426, P11427, P11500, P11833, } \\
\text { P11834, P15137, P3543, P6810, P7491 }\end{array}$ & $\begin{array}{l}\text { New Zealand, Poland, Slovenia, } \\
\text { United Kingdom, United States }\end{array}$ \\
\hline polonica & $P 15005$ & Poland \\
\hline porri & P6207, P7899, P10728, P7518 & Switzerland, Denmark, France, Netherlands \\
\hline primulae & P10220, P10333 & Germany \\
\hline pseudosyringae & P10443, P16355, P10437, P10444, P16354 & Germany, United States \\
\hline pseudotsugae & P10339, $P 10218$ & United States \\
\hline psychrophila & P10434, P10433 & France, Germany \\
\hline quercetorum & P15555 & United States \\
\hline quercina & P10334, P10441 & Germany, Serbia \\
\hline quininea & P3247, $P 8488$ & Peru \\
\hline ramorum & $\begin{array}{l}\text { P10102, P10301, P10084, P10090, P10130, P11047, P11122, P11333, } \\
\text { P10343, P11051 }\end{array}$ & $\begin{array}{l}\text { Germany, Netherlands, United States, Poland, } \\
\text { Belgium }\end{array}$ \\
\hline richardiae & P6875, P3876 & United States \\
\hline rubi & P3316, P3289, P6404, P15596 & $\begin{array}{l}\text { United States, United Kingdom (Scotland), } \\
\text { Germany }\end{array}$ \\
\hline sansomea & $P 3163$ & United States \\
\hline siskiyouensis & P15122, P15123, P16301 & United States \\
\hline sojae & P10704, P3114, P6497, P7061, P0405 & New Zealand, United States, Canada \\
\hline sp. sulawesiensis & P6306 & Indonesia \\
\hline syringae & $\begin{array}{l}\text { P10330, P10332, P2004, P6903, P6901, P11836, P11835, P15090, } \\
\text { P15093, P15092, P15094, P7018, P6208, P3013, P3014, P3016, P3015, } \\
\text { P3012 }\end{array}$ & $\begin{array}{l}\text { Germany, United Kingdom (Scotland), Australia, } \\
\text { Switzerland }\end{array}$ \\
\hline tentaculata & P10363, P8497 & Argentina, Germany \\
\hline
\end{tabular}


TABLE 1. (continued from preceding page)

\begin{tabular}{|c|c|c|}
\hline Species & Isolate number ${ }^{\mathrm{a}}$ & Country \\
\hline sp. thermophilum & $P 10457$ & United States \\
\hline trifolii & $P 1462$ & United States \\
\hline tropicalis & $P 10329$ & United States \\
\hline uliginosa & P10328, P10413 & Germany, Poland \\
\hline vignae & P3019, P7471 & Australia \\
\hline \multicolumn{3}{|l|}{ Outgroup species } \\
\hline Pythium sp. & P8209, P8201 & Ecuador \\
\hline Pythium sylvaticum & $P 15580$ & Canada \\
\hline Phytopythium vexans & P3980 & n.d. ${ }^{\mathrm{d}}$ \\
\hline
\end{tabular}

benzene agar V8 medium (24) for selective isolation of Phytophthora spp. and half used for DNA extraction using the USDAAPHIS protocol (46) with the Qiagen DNeasy Plant MiniKit (Qiagen, Valencia, CA). Cultures were checked after a few days to determine whether a Phytophthora sp. or P. ramorum were growing and with species identification done by conventional morphological classification. DNA extractions were diluted 1/10 in sterile water and tested with the atp9-nad9 Phytophthora genus-specific probe and $P$. ramorum and $P$. kernoviae speciesspecific probes. Results were also independently validated in a PCR hybridization array system (13).

Oregon raspberry: (P. rubi). DNA was extracted from a total of 8 raspberry roots using the previously described USDA-APHIS protocol (46). The DNA was diluted $1 / 10$ and 1/50 and assayed using the atp9-nad9 Phytophthora genus-specific assay with
$P$. rubi and $P$. fragariae species-specific probes. Two DNA samples previously extracted from infected raspberry were also included in the assay.

U.K. samples: (P. kernoviae). DNA from leaves naturally and artificially inoculated with $P$. kernoviae were provided by Jennifer Tomlinson (Food and Environment Research Agency, Sand Hutton, York, UK) and tested in the Martin laboratory using the atp9-nad9 Phytophthora genus-specific assay and the P. ramorum and P. kernoviae species-specific probes.

\section{RESULTS}

Mitochondrial gene order differences in Phytophthora versus Pythium and plants. Three conserved mitochondrial gene order differences were observed when the organization of the

TABLE 2. Pythium and plant species tested with the Phytophthora genus-specific markers and the plant internal control ${ }^{\mathrm{a}}$

\begin{tabular}{|c|c|c|c|c|c|}
\hline Species DNA & Sample number & Phy Genus ATP9 probe & Phy Genus TrnM probe ${ }^{b}$ & Plant probe & ITS SYBR Green $^{c}$ \\
\hline \multicolumn{6}{|l|}{ Pythium $^{\mathrm{d}}$} \\
\hline Pythium oligandrum & $81-10$ & N/A & N/A & N/A & 30.4 \\
\hline P. splendens & $85-3$ & N/A & N/A & N/A & 26.6 \\
\hline$P$. spinosum & $79-4$ & N/A & N/A & N/A & 27.4 \\
\hline P. ultimum (HS isolate) & $23-1$ & N/A & N/A & N/A & 24.9 \\
\hline P. graminicola & 1986-1 & N/A & N/A & N/A & 29.1 \\
\hline P. catenulatum & $1986-8$ & N/A & N/A & N/A & 26.7 \\
\hline P. mamillatum & 1986-37 & N/A & N/A & N/A & 33 \\
\hline P. sylvaticum & $1987-73$ & N/A & N/A & N/A & 30.4 \\
\hline P. paroecandrum & $1987-60$ & N/A & N/A & N/A & 26.1 \\
\hline P. sylvaticum & $1987-14$ & N/A & N/A & N/A & 27.6 \\
\hline P. sulcatum & $1987-98$ & N/A & N/A & N/A & 26.6 \\
\hline P. nunn & $1987-58$ & N/A & N/A & N/A & 28.6 \\
\hline P. myriotylum & $1987-134$ & N/A & N/A & N/A & 25.9 \\
\hline P. ultimum & $1987-69$ & N/A & N/A & N/A & 26.8 \\
\hline P. irregulare & $1987-68$ & N/A & N/A & N/A & 24.6 \\
\hline P. ultimum (HS isolate) & $1987-78$ & N/A & N/A & N/A & 27.5 \\
\hline P. ultimum & $1987-65$ & N/A & N/A & N/A & 26.8 \\
\hline P. ultimum & 1987-92 & N/A & N/A & N/A & 24.4 \\
\hline P. ultimum spor & $1993-25$ & N/A & N/A & N/A & 26 \\
\hline P. aristosporum & $1994-15$ & N/A & N/A & N/A & 21.5 \\
\hline P. volutum & $1995-105$ & N/A & N/A & N/A & 32.5 \\
\hline P. coloratum & 1999-20 & N/A & N/A & N/A & 25.2 \\
\hline P. dissimile & $1999-23$ & N/A & N/A & N/A & 35.7 \\
\hline P. aristosporum & 1999-19 & N/A & N/A & N/A & 32.6 \\
\hline P. vanterpoolii & 1999-31 & N/A & N/A & N/A & 29.7 \\
\hline P. pyrilobum & $1999-28$ & N/A & N/A & N/A & 26.4 \\
\hline P. acanthicum & A-6 & N/A & N/A & N/A & 33.4 \\
\hline P. deliense & 1989-1a & N/A & N/A & N/A & 24.9 \\
\hline P. arrhenomanes & 1991-12 & N/A & N/A & N/A & 26.1 \\
\hline P. irregulare & $1991-15$ & N/A & N/A & N/A & 26.3 \\
\hline \multicolumn{6}{|l|}{ Plant species } \\
\hline Cucumus sativa & $\ldots$ & N/A & N/A & 23.3 & 16.7 \\
\hline \multirow[t]{2}{*}{ Citrullus lantanus } & $\ldots$ & N/A & N/A & 21.1 & 12.9 \\
\hline & & & & & (continued on next page) \\
\hline
\end{tabular}

a $/ \mathrm{A}=$ no amplification and NT $=$ not tested.

${ }^{\mathrm{b}}$ For the TrnM assay, the baseline was 6 to 15 cycles and threshold at 10 .

c Amplification using the internal transcribed spacer (ITS)1 and ITS4 primers (47).

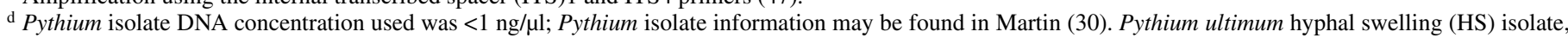
no oospores produced.

e Plant DNA concentration was 0.5 to $16 \mathrm{ng} / \mu \mathrm{l}$.

${ }^{f}$ DNA for plant species from field sample. 
mitochondrial genome of Phytophthora spp. was compared with Pythium spp. and plants. The trnM-trnP-trnM region located between the rRNA-large subunit ( $r n l)$ and ribosomal protein L14 (rpl14) spanned a region of $\approx 258$ bp (size varied among species) containing the three indicated tRNAs (Fig. 1; Table 3). Because of the level of conservation of the tRNA coding sequences, it was possible to design highly conserved forward and reverse primers from the $\operatorname{trn} M$ coding regions (amplicon $\approx 206 \mathrm{bp}$ ) with a genusspecific probe designed from the $3^{\prime}$ end of the first trnM coding region. In total, $>170$ isolates representing a wide range of species in the genus were sequenced and, although there was enough interspecific sequence variation in the coding and spacer sequences to accommodate the design of 38 species-specific probes, this region was not variable enough to use for systematically designing markers for a wide range of Phytophthora spp. and was used subsequently as a genus-specific marker only.

The atp9-nad9 gene order was highly conserved in Phytophthora spp. and the presence of highly conserved sequences allowed for the design of amplification primers in the flanking regions of each gene and a Phytophthora genus-specific probe in the $3^{\prime}$ end of the atp9 gene (Fig. 1; Table 3). Amplicons were $\approx 340 \mathrm{bp}$ (size varied among species) and contained $110 \mathrm{bp}$ of the $3^{\prime}$ end of the atp 9 gene and 85 bp of the nad 9 gene, with the remaining sequences representing intergenic spacer. This region was sequenced for $>720$ isolates representing 91 valid and 30 provisional Phytophthora spp. In total, 84 species-specific probes for Phytophthora were designed from this locus (70 in silico) (Supplementary sequence alignment files).
The nadl1-secY gene order was also highly conserved in Phytophthora spp. but the sequence divergence in these genes precluded the development of conserved primers and probe for genus-specific detection in the coding regions. In the spacer region between these two genes, there is a trnL-trnL tRNA cluster that is suitable for designing a genus-specific marker (amplicon $\approx 172 \mathrm{bp}$ ) and, although there was enough sequence divergence for designing species-specific TaqMan probes for $\approx 65$ species, the same trnL-trnL gene order was present in the Pythium mitochondrial genome and, consequently, a problem with primer specificity leading to background amplification of some Pythium spp. Additional work with this locus was not pursued.

Evaluation of specificity of genus-specific amplification. The trnM-trnM and atp9-nad9 genus-specific primers and probe did not amplify (verified by running amplification on agarose gel) or have any background detection of Pythium or plant species when tested by real-time PCR (Table 2). When evaluated against a wide range of Phytophthora spp. (256 isolates representing 87 valid and 14 provisional Phytophthora spp.; culture accessions in italics in Table 1), the trnM-trnM assay detected all species and isolates (data not shown) while the atp9-nad9 marker also detected all the same isolates and species with the exception of P. bisheria and P. frigida (Table 4).

Specificity of species-specific TaqMan probes. In total, 14 species-specific probes were tested in multiplex with the genusspecific TaqMan probe for specificity against the core plate of Phytophthora spp.; all probes were highly specific and identified only the intended species (Table 4). The probe for the P. citricola

TABLE 2. (continued from preceding page)

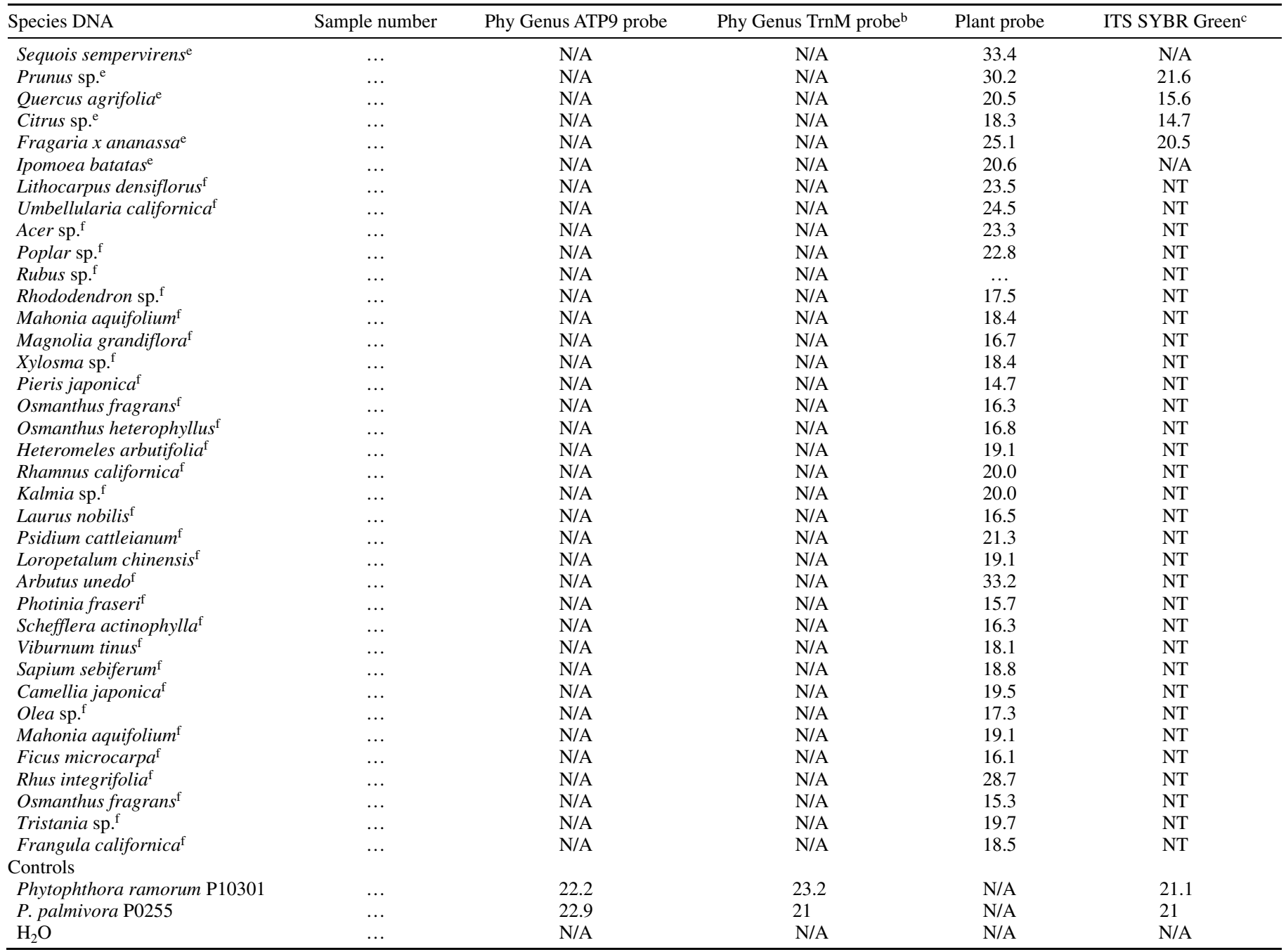


complex detected $P$. citricola, $P$. citricola clade E, $P$. multivora, $P$. pini, and $P$. plurivora. Further testing of the $P$. ramorum and $P$. kernoviae species-specific TaqMan probes was done with a wide range of Phytophthora spp. (256 isolates representing 87 valid and 14 provisional Phytophthora spp.; culture accessions in italics in Table 1) and, in all cases, species specificity was observed. To confirm that the plant internal control would not influence the sensitivity or accuracy of detection of these species, the plant

TABLE 3. Primers and probes tested in this study

\begin{tabular}{|c|c|c|c|c|c|c|c|}
\hline Primer-probe name & Sequence $5^{\prime}-3^{\prime a}$ & Modification & Target & Notes & $\begin{array}{l}\text { Standard curve } \\
\text { equation }^{\text {b }}\end{array}$ & $\begin{array}{c}\mathrm{E} \\
(\%)^{\mathrm{c}}\end{array}$ & $R^{2}$ \\
\hline \multicolumn{8}{|l|}{ Sequencing primers } \\
\hline Nad9-F & TACAACAAGAATTAATGAGAAC & & ATP9-NAD9 spacer & $\cdots$ & $\cdots$ & $\ldots$ & $\ldots$ \\
\hline Nad9-R & GTTAAAATTTGTACTACTAACAT & & ATP9-NAD9 spacer & $\ldots$ & $\ldots$ & $\ldots$ & $\ldots$ \\
\hline Lrn-F & CTGAAAGCATCTAAGTAAGA & & lrn-TrnM region & $\ldots$ & $\ldots$ & $\ldots$ & $\ldots$ \\
\hline TrnM-R & GAACCTACATCTTCAGATTA & & lrn-TrnM region & $\ldots$ & $\ldots$ & $\ldots$ & $\ldots$ \\
\hline PhyG_ATP9_nested_F & TTYTGTTTAATGATGGCTTT & & ATP9-NAD9 spacer & $\begin{array}{l}\text { Nested } \\
\text { primer }\end{array}$ & $\cdots$ & $\cdots$ & $\cdots$ \\
\hline \multicolumn{8}{|l|}{ Real-time PCR primers ${ }^{\mathrm{d}}$} \\
\hline \multicolumn{8}{|l|}{ Genus primers, probe } \\
\hline $\begin{array}{l}\text { ATP9-NAD9 region } \\
\text { PhyG_ATP9_2FTail }\end{array}$ & $\begin{array}{l}\text { ․ } \\
\text { AATAAATCATAACCТТCТTTACAA }\end{array}$ & $\cdots$ & $\cdots$ & $\cdots$ & $y=-3.24 x+18.79$ & 103 & 0.9939 \\
\hline \multirow[t]{2}{*}{ PhyG-R6_Tail } & $\begin{array}{l}\text { CAAGAATTAATG } \\
\text { AATAAATCATAAATACATAATTCA }\end{array}$ & $\cdots$ & Phytophthora sp. & $5^{\prime}$ Flap & $\cdots$ & $\cdots$ & $\cdots$ \\
\hline & $\begin{array}{l}\text { TTTTTATA } \\
\text { AAAGCCATCATTAAACARAATAAA }\end{array}$ & $\cdots$ & Phytophthora sp. & $5^{\prime}$ Flap & $\cdots$ & $\cdots$ & $\cdots$ \\
\hline ATP9_PhyG2_probeR & $\mathrm{GC}$ & Fam/BHQ1 & Phytophthora sp. & Probe & $\ldots$ & $\ldots$ & $\ldots$ \\
\hline TrnM region & & & Phytophthora sp. & $\cdots$ & NA & $\cdots$ & $\cdots$ \\
\hline PhyG-F2 & CGTGGGAATCATAATCCT & $\ldots$ & Phytophthora sp. & $\ldots$ & $\ldots$ & $\ldots$ & $\ldots$ \\
\hline PhyG-Rb & CAGATTATGAGCCTGATAAG & $\ldots$ & Phytophthora sp. & $\ldots$ & $\ldots$ & $\ldots$ & $\ldots$ \\
\hline \multirow[t]{2}{*}{ TrnM_PhyG_probe2 } & ATRTTGTAGGTTCAARTCCTAYCAT & & & & & & \\
\hline & CAT & Hex/BHQ1 & Phytophthora sp. & Probe & $\cdots$ & $\ldots$ & $\ldots$ \\
\hline \multicolumn{8}{|l|}{ Species-specific probes } \\
\hline PfraVf_nad9sp_TaqMan2 & $\begin{array}{l}\text { ATCTCGTAATAGATATATATGTATA } \\
\text { TTTAATACGT }\end{array}$ & Hex/BHQ1 & Phytophthora fragariae & Probe & $y=-3.21 x+19.52$ & 105 & 0.9985 \\
\hline \multirow[t]{2}{*}{ Pcact_nad9sp_probe2 } & TTACATGTTATATAATTATTAACAC & & & & & & \\
\hline & TATTTATAAAA & Quasar670/BHQ2 & P. cactorum & Probe & $y=-3.14 x+20.94$ & 108 & 0.9953 \\
\hline \multirow[t]{2}{*}{ Pker_nad9sp_1Fb } & TTTATATTATTCACAGATTTATTAA & & & & & & \\
\hline & TTTTTTTCTA & Quasar670/BHQ2 & P. kernoviae & Probe & $y=-3.11 x+21.91$ & 110 & 0.9982 \\
\hline \multirow[t]{2}{*}{ Pram_nad9sp_1F } & ACGTTACGTCTAGACTTGTATTATG & & & & & & \\
\hline & CATTG & Hex/BHQ1 & P. ramorum & Probe & $y=-3.21 x+21.56$ & 105 & 0.9727 \\
\hline \multirow[t]{2}{*}{ Palni_nad9sp_probe1 } & AATAGATATATACGTATATTTAAC & & & & & & \\
\hline & GCATAATTAGC & Quasar670/BHQ2 & P. alni & Probe & $y=-2.996 x+22.006$ & 116 & 0.9952 \\
\hline \multirow[t]{2}{*}{ Ppalm_nad9sp_probe2 } & TATAATTACTTAGRCYTGAGTATTT & & & & & & \\
\hline & AAATTGAAA & Quasar670/BHQ2 & $P$. palmivora & Probe & $y=-3.252 x+19.657$ & 103 & 0.996 \\
\hline \multirow[t]{2}{*}{ Psyr_nad9sp_probe1 } & TACTTTTARCTAAATGTTAACTATT & & & & & & \\
\hline & TTTCTAA & Quasar670/BHQ2 & P. syringae & Probe & $y=-2.952 x+21.067$ & 118 & 0.9949 \\
\hline \multirow[t]{2}{*}{ Pcambi_nad9sp_probe1 } & ATCCTATAATAGGTATATATGTAC & & & & & & \\
\hline & ATTTAATGCA & Hex/BHQ1 & P. cambivora & Probe & $y=-3.34 x+22.973$ & 99 & 0.9917 \\
\hline \multirow[t]{2}{*}{ Pcinn_nad9sp_probe1 } & AAGAAATATTTAGTTTATTAATATA & & & & & & \\
\hline & TAATATAACT & Quasar670/BHQ2 & P. cinnamomi & Probe & $y=-3.063 x+20.498$ & 112 & 0.9824 \\
\hline \multirow[t]{2}{*}{ PfraVrubi_Atp9_TaqMan1 } & ATATATACGTGTATTTAATGCATAA & & & & & & \\
\hline & TCAGCTA & Quasar670/BHQ2 & P. rubi & Probe & $y=-3.1631 x+17.334$ & 107 & 0.993 \\
\hline Pcit_nad9sp_T1F & $\begin{array}{l}\text { AATAATAGTTTATTTTTTTGATATA } \\
\text { TAAATATTTAT }\end{array}$ & $\begin{array}{l}\text { CALFluorRed } \\
\text { 610/BHQ2 }\end{array}$ & P. citricola group & Probe & $y=-3.043 x+22.058$ & 113 & 0.9936 \\
\hline Pnicot_ATP9_Probe1 & ATGTTATATCATTATTTTTTATTAT & & & & & & \\
\hline & ATATACAAAT & Quasar670/BHQ2 & P. nicotianae & Probe & NA & $\ldots$ & $\ldots$ \\
\hline Ppssyr_ATP9_Probe & TTAGATATGTAAGTACTTATAGTGT & & & & & & \\
\hline & TTATATT & Quasar670/BHQ2 & P. pseudosyringae & Probe & $y=-3.362 x+20.457$ & 98 & 0.9918 \\
\hline Pgona_nad9sp_probe2 & $\begin{array}{l}\text { ATAATACACGTATACTTAAACCCTT } \\
\text { TTTAGTA }\end{array}$ & Quasar670/BHQ2 & P. lacustris & $\begin{array}{l}\text { Probe, } \\
\text { Salix-soil }\end{array}$ & $y=-3.546 x+17.936$ & 91 & 0.9984 \\
\hline Plant primers and probe & & & & & & & \\
\hline FMPl2b & GCGTGGACCTGGAATGACTA & $\ldots$ & Plant & $\ldots$ & $\ldots$ & $\ldots$ & $\ldots$ \\
\hline FMPl3b & AGGTTGTATTAAAGTTTCGATCG & $\ldots$ & Plant & $\ldots$ & $\ldots$ & $\ldots$ & $\ldots$ \\
\hline Plant CAL-Red probe & $\begin{array}{l}\text { CTTTTATTATCACTTCCGGTACTGG } \\
\text { CAGG }\end{array}$ & $\begin{array}{l}\text { CALFluorRed } \\
610 / \mathrm{BHQ} 2\end{array}$ & Plant & Probe & NA & $\ldots$ & $\ldots$ \\
\hline ITS universal primers ${ }^{\mathrm{f}}$ & & & & & & & \\
\hline ITS1g & TCCGTAGGTGAACCTGCGG & $\ldots$ & Universal & SYBR & & & \\
\hline & & & & Green & $\ldots$ & $\cdots$ & $\cdots$ \\
\hline ITS4 $4^{g}$ & TCCTCCGCTTATTGATATGC & $\ldots$ & Universal & SYBR & & & \\
\hline & & & & Green & $\ldots$ & $\cdots$ & $\ldots$ \\
\hline
\end{tabular}

a $=$ not available.

b Sequences in bold at the start of the amplification primers are the $5^{\prime}$ flaps, as described by Afonina et al. (1) and Arif et al. (2).

c Efficiency.

${ }^{\mathrm{d}} \mathrm{PCR}=$ polymerase chain reaction.

e Tooley et al. (45).

f Internal transcribed spacer (ITS) universal primers used with SYBR Green.

g White et al. (47). 


\begin{tabular}{|c|c|c|c|c|c|c|c|c|c|c|c|c|c|c|c|}
\hline & 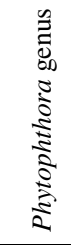 & $\begin{array}{l}3 \\
\bar{\Xi} \\
\text { Q }\end{array}$ & 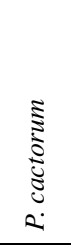 & 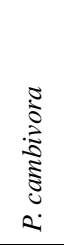 & 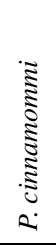 & 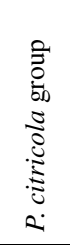 & 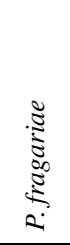 & 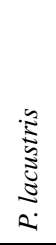 & 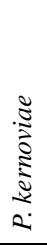 & 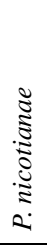 & 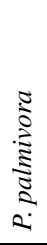 & 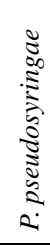 & 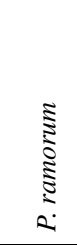 & 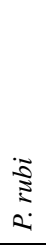 & 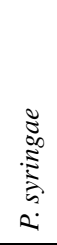 \\
\hline Target probes tested $\mathrm{a}^{\mathrm{a}}$ & 57 & 60 & 57 & 60 & 57 & 60 & 57 & 57 & 57 & 53 & 57 & 57 & 57 & 60 & 60 \\
\hline \multicolumn{16}{|l|}{ Phytophthora spp. } \\
\hline$a l n i^{\mathrm{d}}$ & 22.8 & 23.9 & - & - & - & - & - & - & - & - & - & - & - & - & - \\
\hline andina & 20.9 & - & - & - & - & - & - & - & - & - & - & - & - & - & - \\
\hline asparagi n. $i^{\mathrm{d}}$ & 21.1 & - & - & - & - & - & - & - & - & - & - & - & - & - & - \\
\hline austrocedrae $e^{\mathrm{d}}$ & 21.2 & - & - & - & - & - & - & - & - & - & - & - & - & - & - \\
\hline bisheria $^{\mathrm{d}}$ & - & - & - & - & - & - & - & - & - & - & - & - & - & - & - \\
\hline boehmeriae $^{\mathrm{d}}$ & 20.3 & - & - & - & - & - & - & - & - & - & - & - & - & - & - \\
\hline botryosa $^{\mathrm{d}}$ & 21.4 & - & - & - & - & - & - & - & - & - & - & - & - & - & - \\
\hline brassicae $^{\mathrm{d}}$ & 21.2 & - & - & - & - & - & - & - & - & - & - & - & - & - & - \\
\hline cactorum $^{\mathrm{d}}$ & 22.7 & - & 21.9 & - & - & - & - & - & - & - & - & - & - & - & - \\
\hline cajani & 24.2 & - & - & - & - & - & - & - & - & - & - & - & - & - & - \\
\hline cambivora $^{\mathrm{d}}$ & 25.4 & - & - & 26.9 & - & - & - & - & - & - & - & - & - & - & - \\
\hline sp. canalensis & 22.2 & - & - & - & - & - & - & - & - & - & - & - & - & - & - \\
\hline capsici $^{\mathrm{d}}$ & 21.9 & - & - & - & - & - & - & - & - & - & - & - & - & - & - \\
\hline captiosa $^{\mathrm{d}}$ & 29 & - & - & - & - & - & - & - & - & - & - & - & - & - & - \\
\hline castaneae $^{\mathrm{d}}$ & 21.5 & - & - & - & - & - & - & - & - & - & - & - & - & - & - \\
\hline \multicolumn{16}{|l|}{ cinnamomi } \\
\hline var. robiniae ${ }^{\mathrm{d}}$ & 19.7 & - & - & - & - & - & - & - & - & - & - & - & - & - & - \\
\hline citricola complex ${ }^{\mathrm{d}}$ & 20.2 & - & - & - & - & 20.9 & - & - & - & - & - & - & - & - & - \\
\hline citrophthora ${ }^{\mathrm{d}}$ & 21.1 & - & - & - & - & - & - & - & - & - & - & - & - & - & - \\
\hline clandestina $^{\mathrm{d}}$ & 22.3 & - & - & - & - & - & - & - & - & - & - & - & - & - & - \\
\hline colocasiae $^{\mathrm{d}}$ & 28.7 & - & - & - & - & - & - & - & - & - & - & - & - & - & - \\
\hline cryptogea $^{\mathrm{d}}$ & 21.7 & - & - & - & - & - & - & - & - & - & - & - & - & - & - \\
\hline cuyabensis $^{\mathrm{d}}$ & 19.5 & - & - & - & - & - & - & - & - & - & - & - & - & - & - \\
\hline drechsleri $^{\mathrm{d}}$ & 24 & - & - & - & - & - & - & - & - & - & - & - & - & - & - \\
\hline erythrosepticad & 22.5 & - & - & - & - & - & - & - & - & - & - & - & - & - & - \\
\hline fallax $^{\mathrm{d}}$ & 32.6 & - & - & - & - & - & - & - & - & - & - & - & - & - & - \\
\hline foliorum $^{\mathrm{d}}$ & 22.9 & - & - & - & - & - & - & - & - & - & - & - & - & - & - \\
\hline fragariae ${ }^{\mathrm{d}}$ & 19.4 & - & - & - & - & - & 18.9 & - & - & - & - & - & - & - & - \\
\hline frigida & - & - & - & - & - & - & - & - & - & - & - & - & - & - & - \\
\hline glovera $^{\mathrm{d}}$ & 22.3 & - & - & - & - & - & - & - & - & - & - & - & - & - & - \\
\hline gonapodyides ${ }^{\mathrm{c}, \mathrm{d}}$ & 25.9 & - & - & - & - & - & - & - & - & - & - & - & - & - & - \\
\hline hedraiandra ${ }^{\mathrm{d}}$ & 22.9 & - & - & - & - & - & - & - & - & - & - & - & - & - & - \\
\hline hevea $^{\mathrm{d}}$ & 21.3 & - & - & - & - & - & - & - & - & - & - & - & - & - & - \\
\hline hibernalis $^{\mathrm{d}}$ & 22.5 & - & - & - & - & - & - & - & - & - & - & - & - & - & - \\
\hline humicola $^{\mathrm{d}}$ & 21.8 & - & - & - & - & - & - & - & - & - & - & - & - & - & - \\
\hline idaei & 23.2 & - & - & - & - & - & - & - & - & - & - & - & - & - & - \\
\hline ilicis $^{\mathrm{d}}$ & 25.9 & - & - & - & - & - & - & - & - & - & - & - & - & - & - \\
\hline infestans $^{\mathrm{d}}$ & 23.1 & - & - & - & - & - & - & - & - & - & - & - & - & - & - \\
\hline insolita $^{\mathrm{d}}$ & 26.2 & - & - & - & - & - & - & - & - & - & - & - & - & - & - \\
\hline inundata $^{\mathrm{d}}$ & 22.6 & - & - & - & - & - & - & - & - & - & - & - & - & - & - \\
\hline ipomoea $^{\mathrm{d}}$ & 21.9 & - & - & - & - & - & - & - & - & - & - & - & - & - & - \\
\hline iranica & 23.2 & - & - & - & - & - & - & - & - & - & - & - & - & - & - \\
\hline kelmania $^{\mathrm{d}}$ & 22.6 & - & - & - & - & - & - & - & - & - & - & - & - & - & - \\
\hline kernoviae $^{\mathrm{d}}$ & 23.3 & - & - & - & - & - & - & - & 23 & - & - & - & - & - & - \\
\hline lacrimae $^{\mathrm{d}}$ & 20.5 & - & - & - & - & - & - & - & - & - & - & - & - & - & - \\
\hline lagoariana $^{\mathrm{d}}$ & 22.8 & - & - & - & - & - & - & - & - & - & - & - & - & - & - \\
\hline 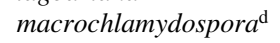 & 23.2 & - & - & - & - & - & - & - & - & - & - & - & - & - & - \\
\hline meadii & 21.1 & - & - & - & - & - & - & - & - & - & - & - & - & - & - \\
\hline medicaginis $^{\mathrm{d}}$ & 30.3 & - & - & - & - & - & - & - & - & - & - & - & - & - & - \\
\hline megakarya ${ }^{\mathrm{d}}$ & 22.1 & - & - & - & - & - & - & - & - & - & - & - & - & - & - \\
\hline megasperma $a^{\mathrm{d}}$ & 20.2 & - & - & - & - & - & - & - & - & - & - & - & - & - & - \\
\hline melonis $^{\mathrm{d}}$ & 21.1 & - & - & - & - & - & - & - & - & - & - & - & - & - & - \\
\hline mengei & 21.4 & - & - & - & - & - & - & - & - & - & - & - & - & - & - \\
\hline mirabilis ${ }^{\mathrm{d}}$ & 21.4 & - & - & - & - & - & - & - & - & - & - & - & - & - & - \\
\hline \multirow[t]{2}{*}{ multivesiculata } & 22 & - & - & - & - & - & - & - & - & - & - & - & - & - & - \\
\hline & & & & & & & & & & & & & \multicolumn{3}{|c|}{ (continued on next page) } \\
\hline
\end{tabular}

a All baselines were set up at 8 to 16 cycles, excepted for $P$. fragariae (6 to 18); P. pseudosyringae and P. ramorum were "auto" set up. The threshold was set at 50 for all assays excepted for $P$. cactorum $\left(\mathrm{C}_{\mathrm{t}}=25\right)$, P. kernoviae $\left(\mathrm{C}_{\mathrm{t}}=100\right)$, and P. palmivora $\left(\mathrm{C}_{\mathrm{t}}=75\right)$.

$\mathrm{b}$ The DNA preparation could be amplified with primers for other loci but - indicates that these species were not detected by this genus-specific primer and probe combination.

${ }^{\mathrm{c}}$ Not tested with the core plate but tested with other isolates: P. lateralis P3361; P. lacustris P10337, P10283, and P10284; P. mexicana P0646; Pythium sp. P8209 and P8201; and P. megasperma P1679.

${ }^{d}$ Tested again with all isolates of this specific species and closely related species with the same results for specificity. 
Cycle threshold $\left(\mathrm{C}_{\mathrm{t}}\right)$ value at each annealing temperature $\left({ }^{\circ} \mathrm{C}\right)^{\mathrm{b}}$

\begin{tabular}{|c|c|c|c|c|c|c|c|c|c|c|c|c|c|c|c|}
\hline & 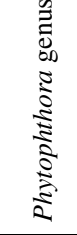 & $\begin{array}{l}\tilde{\Xi} \\
\text { 2 } \\
\end{array}$ & 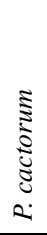 & 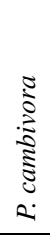 & 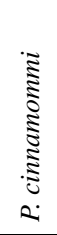 & 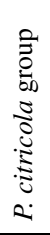 & 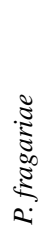 & 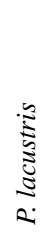 & 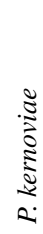 & 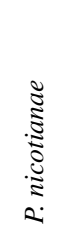 & 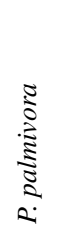 & 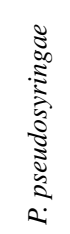 & 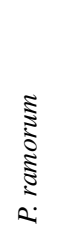 & 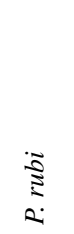 & 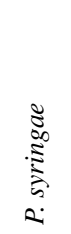 \\
\hline Target probes tested ${ }^{\mathrm{a}}$ & 57 & 60 & 57 & 60 & 57 & 60 & 57 & 57 & 57 & 53 & 57 & 57 & 57 & 60 & 60 \\
\hline napoensis ${ }^{\mathrm{d}}$ & 24 & - & - & - & - & - & - & - & - & - & - & - & - & - & - \\
\hline nemorosa $a^{\mathrm{d}}$ & 29.3 & - & - & - & - & - & - & - & - & - & - & - & - & - & - \\
\hline nicotianae $^{\mathrm{d}}$ & 27.7 & - & - & - & - & - & - & - & - & 22.6 & - & - & - & - & - \\
\hline niederhauserii ${ }^{\mathrm{d}}$ & 21.3 & - & - & - & - & - & - & - & - & - & - & - & - & - & - \\
\hline novaeguineae $e^{\mathrm{d}}$ & 18.8 & - & - & - & - & - & - & - & - & - & - & - & - & - & - \\
\hline ohioensis & 22.2 & - & - & - & - & - & - & - & - & - & - & - & - & - & - \\
\hline palmivora $^{\mathrm{d}}$ & 20.2 & - & - & - & - & - & - & - & - & - & 21 & - & - & - & - \\
\hline parsiana $^{\mathrm{d}}$ & 22.1 & - & - & - & - & - & - & - & - & - & - & - & - & - & - \\
\hline parvispora $^{\mathrm{d}}$ & 26.7 & - & - & - & - & - & - & - & - & - & - & - & - & - & - \\
\hline personii & 22 & - & - & - & - & - & - & - & - & - & - & - & - & - & - \\
\hline phaseoli $^{\mathrm{d}}$ & 23.9 & - & - & - & - & - & - & - & - & - & - & - & - & - & - \\
\hline pinifolia & 21.5 & - & - & - & - & - & - & - & - & - & - & - & - & - & - \\
\hline pistaciae $^{\mathrm{d}}$ & 20.1 & - & - & - & - & - & - & - & - & - & - & - & - & - & - \\
\hline polonica & 32.7 & - & - & - & - & - & - & - & - & - & - & - & - & - & - \\
\hline porri $^{\mathrm{d}}$ & 29 & - & - & - & - & - & - & - & - & - & - & - & - & - & - \\
\hline primulae $^{\mathrm{d}}$ & 21.9 & - & - & - & - & - & - & - & - & - & - & - & - & - & - \\
\hline pseudosyringae $e^{\mathrm{d}}$ & 22.4 & - & - & - & - & - & - & - & - & - & - & 21.8 & - & - & - \\
\hline pseudotsugae $^{\mathrm{d}}$ & 22 & - & - & - & - & - & - & - & - & - & - & - & - & - & - \\
\hline psychrophila ${ }^{\mathrm{d}}$ & 21.1 & - & - & - & - & - & - & - & - & - & - & - & - & - & - \\
\hline quercetorum $\mathrm{d}^{\mathrm{d}}$ & 22.1 & - & - & - & - & - & - & - & - & - & - & - & - & - & - \\
\hline quercina $^{\mathrm{d}}$ & 24.4 & - & - & - & - & - & - & - & - & - & - & - & - & - & - \\
\hline quininea $^{\mathrm{d}}$ & 23.1 & - & - & - & - & - & - & - & - & - & - & - & - & - & - \\
\hline ramorum ${ }^{\mathrm{d}}$ & 22.3 & - & - & - & - & - & - & - & - & - & - & - & 25 & - & - \\
\hline richardiae $^{\mathrm{d}}$ & 22.4 & - & - & - & - & - & - & - & - & - & - & - & - & - & - \\
\hline$r u b i^{\mathrm{d}}$ & 21.2 & - & - & - & - & - & - & - & - & - & - & - & - & 20.2 & - \\
\hline sansomea & 19.3 & - & - & - & - & - & - & - & - & - & - & - & - & - & - \\
\hline sinensis $^{\mathrm{d}}$ & 23.6 & - & - & - & - & - & - & - & - & - & - & - & - & - & - \\
\hline siskiyouensis $^{\mathrm{d}}$ & 20.3 & - & - & - & - & - & - & - & - & - & - & - & - & - & - \\
\hline sojae $^{\mathrm{d}}$ & 24.9 & - & - & - & - & - & - & - & - & - & - & - & - & - & - \\
\hline sulawesiensis & 21.1 & - & - & - & - & - & - & - & - & - & - & - & - & - & - \\
\hline syringae $^{\mathrm{d}}$ & 22.8 & - & - & - & - & - & - & - & - & - & - & - & - & - & 23.7 \\
\hline tentaculata ${ }^{\mathrm{d}}$ & 35.7 & - & - & - & - & - & - & - & - & - & - & - & - & - & - \\
\hline thermophilum & 19.1 & - & - & - & - & - & - & - & - & - & - & - & - & - & - \\
\hline trifolii & 22.7 & - & - & - & - & - & - & - & - & - & - & - & - & - & - \\
\hline tropicalis & 27.3 & - & - & - & - & - & - & - & - & - & - & - & - & - & - \\
\hline uliginosa $^{\mathrm{d}}$ & 20.3 & - & - & - & - & - & - & - & - & - & - & - & - & - & - \\
\hline vignae $^{\mathrm{d}}$ & 22.2 & - & - & - & - & - & - & - & - & - & - & - & - & - & - \\
\hline Pythium sylvaticum & - & - & - & - & - & - & - & - & - & - & - & - & - & - & - \\
\hline Phytopythium vexans & - & - & - & - & - & - & - & - & - & - & - & - & - & - & - \\
\hline
\end{tabular}

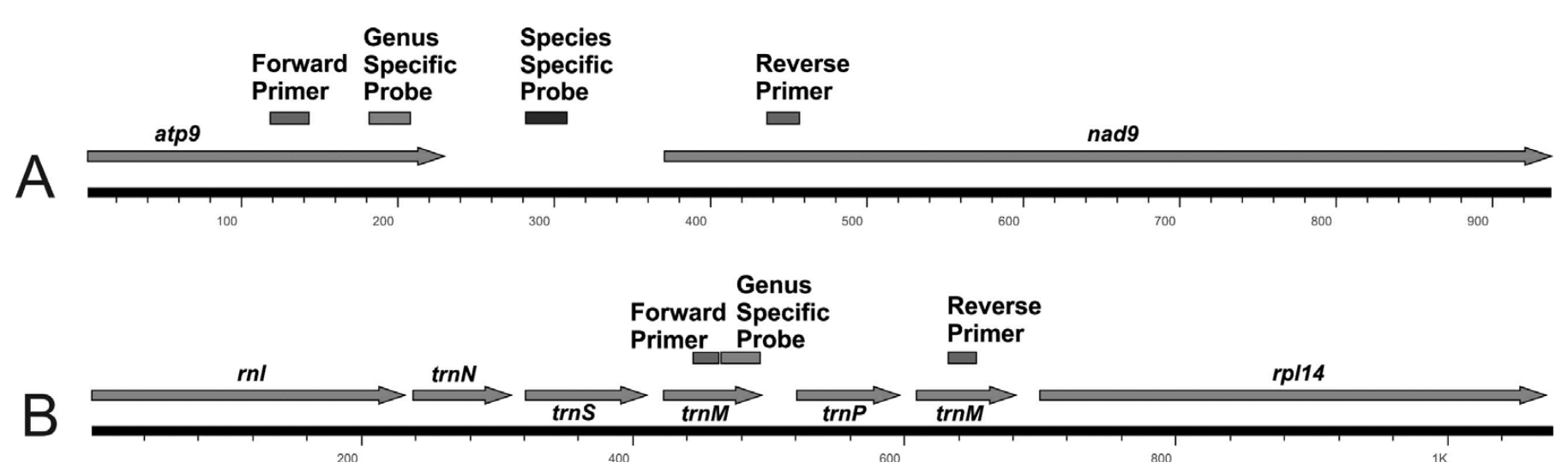

Fig. 1. Phytophthora genus-specific primer and probe location on mitochondrial genome: A, atp9-nad 9 region and $\mathbf{B}$, rnl-rpll4 region.

amplification primers and TaqMan probe were included in these assays and had no effect on results. When tested against additional isolates of the species the probe was designed to detect, as well as other species with sequence similarity at the probe an- nealing site, the 12 remaining species-specific TaqMan probes were all species specific (Table 5). This includes the probe specific for the hybrid species $P$. alni, which detected all three subspecies ( $P$. alni subspp. alni, multiformis, and uniformis). 
Details on specific isolates included in this analysis may be found in Supplementary Table 1a to $\mathrm{k}$, repectively $(P$. ramorum and $P$. kernoviae, $P$. alni, $P$. cactorum and $P$. fragariae, $P$. cambivora and $P$. syringe, $P$. citricola (group), $P$. cinnamomi, $P$. nicotianae, $P$. palmivora, $P$. pseudosyringae, $P$. rubi, and $P$. lacustris). Some of these additional validations were run in multiplex to mimic how the markers may be used in diagnostic labs. The tests for $P$. ramorum and $P$. kernoviae were run together with the atp9nad9 Phytophthora genus-specific probe. To mimic detection of the pathogen in strawberry, $P$. cactorum, $P$. fragariae, and the $P$. citricola complex probes were multiplexed. To mimic detection of a Phytophthora sp. in forest or nurseries samples, probes for $P$. syringae and $P$. cambivora were multiplexed.

Standard curve. To evaluate amplification efficiency and the limit of detection, a standard curve for all genus- and speciesspecific assays was determined using a dilution series of target DNA $\left[\mathrm{E}=\left(10^{(-1 / \text { slope })}-1\right) \times 100\right]$ (Table 2). Amplification efficiency was usually close to or slightly greater than $100 \%$. In general, amplification of the Phytophthora genus-specific atp9-nad9 assay exhibited a linear response until the DNA concentration was $<100$ fg $\left(10^{-4}\right.$ dilution $)$, with an amplification efficiency of $103 \%$.

Marker validation with field samples. The two genus-specific amplicons and six of the atp9-nad9 species-specific probes were evaluated with 99 field samples collected by the CDFA in Sacramento, CA (Table 6). In all, 16 different taxonomic entities were identified (11 described species and five unnamed phylogenetic species) in a total of 58 samples; no Phytophthora sp. was detected with either genus-specific marker in 41 of the samples. These results agreed with the findings of the CDFA (based on culturing of the isolate or a positive PCR using the accepted USDA-APHIS $P$. ramorum molecular assay). All probes were confirmed to lack nonspecific background detection with these environmental samples and the species specificity for four of the probes was validated; $P$. ramorum was accurately detected in 11 samples with no false positives for the four $P$. hibernalis and three $P$. foliorum samples; $P$. pseudosyringae was detected in three samples with no false positive for the $P$. nemorosa sample; and $P$. syringae was detected in 11 samples with no false positive for the $P$. austrocedrae sample. Three additional samples tested positive for $P$. syringae but these had a sequence identity of $97 \%$ when the sequence of the atp9-nad9 genus-specific amplicon was compared with known $P$. syringae isolates. The species-specific probe for the $P$. citricola complex detected $P$. multivora in five samples, $P$. pini in one sample, and $P$. plurivora in three samples. The only species-specific probes that were not validated in these environmental samples were for $P$. kernoviae and $P$. cambivora (all samples were negative for these species).

In addition to formally described species, several undescribed phylogenetic species were detected. $P$. sp. aff. colocasiae 1 (three samples) is a phylogenetic species closely related to $P$. colocasiae but distinct in phylogenetic analysis (F. Martin, unpublished data). Likewise, $P$. sp. aff. brassicae 1 (two samples from Schefflera actinophylla) is another phylogenetic species closely related to $P$. brassicae. Two species were also detected that did not match any of the other sequences in our database or GenBank (samples 1518453-1 and 1556826) and may represent new, undescribed species.

Analysis of the 18 samples collected from Big Sur with the atp9-nad9 genus-specific and $P$. ramorum and $P$. kernoviae species-specific probes revealed five samples that were culture positive for $P$. ramorum, all of which were also positive with the genus- and species-specific $P$. ramorum probe (Table 7). One sample that was culture negative had a positive real-time PCR result (sample 6) with a relatively low cycle threshold $\left(\mathrm{C}_{\mathrm{t}}\right)$ of 26.6. No positive detections were observed for the P. kernoviae probe. Two additional samples were tested but, because the plant positive control did not amplify, they were not included in the analysis (data not shown). Of the 10 root samples from Rubus spp., 7 of them tested positive for $P$. rubi with both the trnM-trnM and atp9-nad9 genus-specific markers. All of these samples also tested positive with the atp9-nad9 $P$. rubi probe (Table 8). None of these samples tested positive with the $P$. fragariae probe, confirming the species specificity of detection of this probe. Of the five samples extracted from tissue infected with $P$. kernoviae, four tested positive with the atp9-nad9 genus-specific and $P$. kernoviae-specific probe and one tested positive for P. ramorum (data not shown). These results agreed with the collaborator's identifications the samples.

\section{DISCUSSION}

Comparative mitochondrial genomics was effective for identifying gene order differences useful for developing genus- and species-specific markers for Phytophthora. With this approach, we hoped to improve the specificity of the detection assay and provide a technique that would work under a wider range of amplification conditions with a reduced risk of false positives, the thought being that, even if nonspecific primer annealing occurred due to lower stringency (such as reduced annealing temperatures), the intervening distance between the primers would be too great for amplification to occur under the cycling parameters used. The loci that were the primary focus of this investigation had primer annealing sites that were close enough in Phytophthora spp. to be useful for designing a TaqMan real-time PCR assay but distant enough $(10 \mathrm{~kb}+)$ in the related genus Pythium and plant species to prevent amplification.

Three regions exhibiting conserved gene order differences in the genus Phytophthora were evaluated for developing primers

TABLE 5. Listing of species and number of isolates on which the Phytophthora primers and species-specific TaqMan probes were tested on to fully validate specificity

\begin{tabular}{|c|c|}
\hline Species-specific probe ${ }^{a}$ & Species (number of isolates) tested ${ }^{b}$ \\
\hline Phytophthora alni & P. alni subsp. alni (7), subsp. multiformis (1), subsp. uniformis (2), P. fragariae (19), P. rubi (4) \\
\hline P. cactorum & P. alni subsp. alni (2), subsp. multiformis (1), subsp. uniformis (1), P. cactorum (48), P. fragariae (20), P. rubi (5), P. hedrianda (11) \\
\hline P. cambivora & P. cambivora $(15)$, P. syringae $(18)$ \\
\hline P. cinnamomi & P. cinnamomi (60), P. parvispora (5), P. cinnamomi var. robinae (2), P. sp. niederhauseri $(7)$ \\
\hline P. citricola group & $\begin{array}{l}\text { P. citricola (7), P. citricola clade E (3), P. multivora (8), P. pini (4), P. plurivora (23), P. citrophthora (1), P. europea (3), } \\
\text { P. lateralis (3), P. mengei (3), P. mexicana (1) }\end{array}$ \\
\hline P. fragariae & P. alni subsp. alni (2), subsp. multiformis (1), subsp. uniformis (1), P. cactorum (53), P. fragariae (20), P. rubi (5), P. hedrianda (8) \\
\hline P. lacustris & P. lacustris (3), P. gonapodyides (24), P. sp. PgChlamydo (5) \\
\hline P. nicotianae & P. nicotianae (46), P. infestans (2), P. cactorum (1), P. ipomoeae (1), P. mirabilis (2), P. phaseoli (2) \\
\hline P. palmivora & P. palmivora $(52), P$. megakarya $(20)$ \\
\hline P. pseudosyringae & P. pseudosyringae (4) \\
\hline P. rubi & P. alni subsp. alni (7), subsp. multiformis (1), subsp. uniformis (2), P. fragariae (19), P. rubi (4) \\
\hline P. syringae & P. cambivora (15), P. syringae (18) \\
\hline
\end{tabular}

${ }^{\text {a }}$ Probes for P. ramorum and P. kernoviae were tested against 231 isolates in italics in Table 1 representing 87 described and 14 provisional Phytophthora spp.

${ }^{b}$ Isolates used in this validation were different from the core plate of isolates tested in Table 4 unless only a single representative isolate was available. 
and probes for multiplex real-time PCR detection: atp9-nad9, trnM-trnM, and nadl1-secY. With the exception of the nadl1$\sec Y$ locus, the other loci had highly conserved sequences in the flanking genes for design of amplification primers and exhibited polymorphisms in the intervening spacer sequences useful for design of species-specific TaqMan probes. The atp9-nad9 region was the most useful region because the amplicon size of $\approx 340 \mathrm{bp}$ was suitable for TaqMan real-time PCR, a single primer pair was used to generate an amplicon where both a genus and speciesspecific probe could anneal, and the intergenic spacer region exhibited enough sequence divergence that species-specific probes could be designed for the widest range of species. Unfortunately, the genus-specific primer pair was unable to amplify $P$. bisheria and $P$. frigida. Although the reason for this has not been determined conclusively, preliminary data suggest that there is an unusual gene order difference in these species that separates the two target genes (T. Miles and F. Martin, unpublished data).
The second region examined spanned three tRNAs (trnM-trnPtrnM) located between the $r n l$ and $r p l l 4$ coding regions. A single primer pair generated an amplicon from all species of $\approx 206 \mathrm{bp}$ that contained annealing sites for a Phytophthora genus-specific probe and, for some species, a species-specific probe. Speciesspecific probes could be designed for approximately half the number of species as the atp9-nad9 locus due to a lower level of sequence divergence than the 340-bp amplicon. For this reason, work with this locus focused only on developing an additional Phytophthora genus-specific detection assay. The nad11-secY region was the third region examined and, due to the inability to design truly genus-specific primers, work using this locus was discontinued.

The atp9-nad9 and trnM-trnM markers were validated for genus specificity by testing against 29 Pythium spp. and 39 plant species as well as a total of 256 isolates representing 87 described and 14 provisional Phytophthora spp. Consistent results were also

TABLE 6. Results for real-time polymerase chain reaction (PCR) assays with environmental samples collected from California and tested with Phytophthora genus- and species-specific probes for Phytophthora ramorum, P. kernoviae, P. syringae, P. cambivora, $P$. pseudosyringae, and the $P$. citricola group

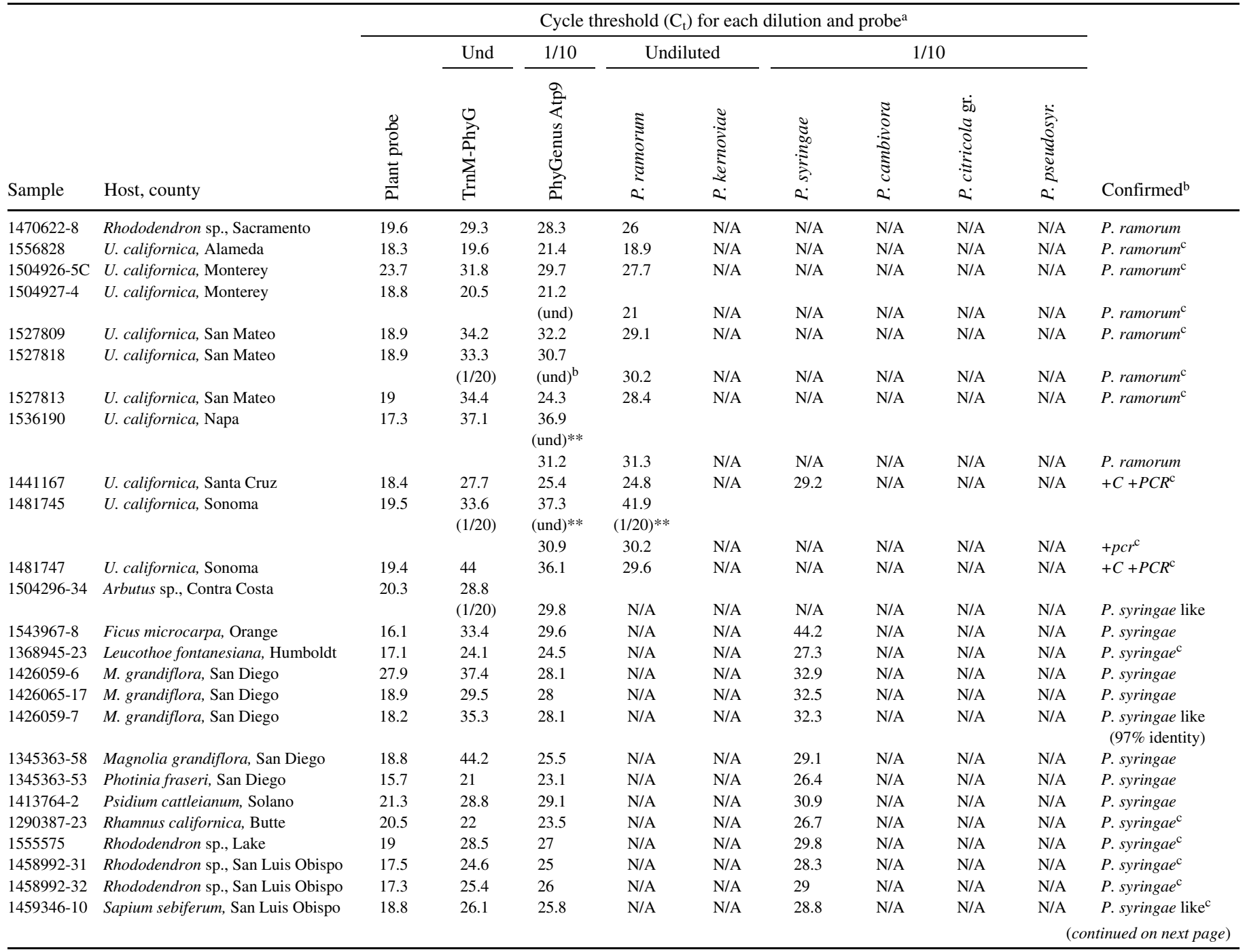

${ }^{a}$ Numbers in parentheses indicate that different amounts of DNA were used in the amplification than indicated in the column heading. A $1 / 20$ refers to using this dilution rather than the $1 / 10$ and und refers to using undiluted DNA. In some cases (**), two $C_{t}$ values with one of the alternative DNA concentrations previously mentioned are listed; in these examples, two different DNA dilutions were tested. N/A = no amplification and PS $=$ poor sequence data, unable to determine species.

b Sequence confirmed. Genus-specific amplicons were sequenced and the results compared against a sequence database generated from DNA from purified cultures of all isolates listed in Table 1 .

${ }^{\mathrm{c}}$ Species identification was confirmed by culturing the pathogen or a positive amplification with the USDA-APHIS-approved P. ramorum molecular diagnostic assay. Cultures were not obtained for all samples.

${ }^{\mathrm{d}}$ Based on DNA sequence analysis of the $\operatorname{cox} 2$, nad $9, \operatorname{rps} 10$, and $\sec Y$ genes, isolates representing this group are phylogenetically distinct and are closely related to the indicated formally described species (F. Martin, unpublished data). 
observed between the two different markers when used to evaluate the presence of a Phytophthora sp. in the 130 environmental samples evaluated. In an effort to improve the fluorescent signal of the atp9-nad9 detection and, hence, the sensitivity of the assay, amplification primers were modified with a $5^{\prime}$ flap (tail of AAT AAATCATAA) that did not anneal to target sequences in a similar fashion as reported by Afonina et al. (1) and Arif et al. (2). Using these modified primers improved the amplification efficiency of the reaction, increased the fluorescence signal compared with using unmodified primers, and thereby increased sensitivity of detection.

Due to the level of sequence divergence observed in the spacer region between the atp 9 and nad 9 genes, this region was useful for the systematic design of species-specific TaqMan probes that could be multiplexed with the atp9-nad9 genus-specific assay. Species-specific probes were validated for 13 species, including the quarantine pathogens $P$. ramorum and $P$. kernoviae, and one species complex. As part of the validation process, specificity was tested against (i) multiple isolates of the target species collected from different geographic regions, (ii) other species that exhibited sequence similarity at the probe annealing site, and (iii) environmental samples. After the work on the specific primer for the $P$. citricola complex was validated in this study, this species complex has been separated into P. citricola, P. multivora (42), P. plurivora (25), P. capensis (3), P. pini (21), and P. mengei (22); consequently, the TaqMan probe is specific only for the species complex. The genus-specific amplicon was sequenced for $>720$ isolates representing 114 species (90 recognized, 17 provisional, and 7 phylogenetically distinct species) (F. Martin, unpublished data). In silico analysis of this data reveled sequence differences that should support the development of TaqMan probes specific

\begin{tabular}{|c|c|c|c|c|c|c|c|c|c|c|c|}
\hline \multirow[b]{3}{*}{ Sample } & \multirow[b]{3}{*}{ Host, county } & \multicolumn{9}{|c|}{ Cycle threshold $\left(\mathrm{C}_{\mathrm{t}}\right)$ for each dilution and probe ${ }^{\mathrm{a}}$} & \multirow[b]{3}{*}{ Confirmed ${ }^{\mathrm{b}}$} \\
\hline & & \multirow[b]{2}{*}{$\begin{array}{l}0 \\
0 \\
0 \\
\vec{a} \\
\frac{\vec{E}}{a}\end{array}$} & \multirow{2}{*}{ Und } & \multirow{2}{*}{ 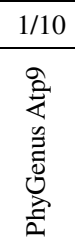 } & \multicolumn{2}{|c|}{ Undiluted } & \multicolumn{4}{|c|}{$1 / 10$} & \\
\hline & & & & & 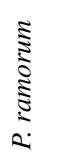 & 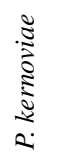 & 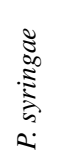 & 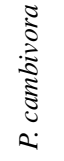 & 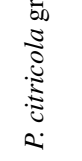 & 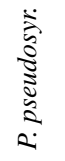 & \\
\hline $1353896-26$ & L. chinensis, Los Angeles & 20.5 & 42.4 & $\begin{array}{r}29.4 \\
\text { (und) }\end{array}$ & N/A & N/A & N/A & N/A & $\begin{array}{c}37.7 \\
\text { (und) }\end{array}$ & N/A & P. multivora \\
\hline $1353896-20$ & Loropetalum chinensis, Los Angeles & 19.1 & 43.3 & 31 & N/A & N/A & N/A & N/A & 33.4 & N/A & P. multivora \\
\hline $1404765-4$ & Osmanthus heterophyllus, Sacramentc & 16.8 & 21.8 & 23 & N/A & N/A & N/A & N/A & 26.6 & N/A & P. multivora \\
\hline 1543960-14 & Rhododendron sp., Orange & 28.7 & 26.5 & 25.3 & N/A & N/A & N/A & N/A & 29.7 & N/A & P. multivora \\
\hline $1503028-1$ & U. californica, Contra Costa & 18.1 & 21.6 & 22.8 & N/A & N/A & 30.6 & N/A & 28.7 & N/A & P. multivora \\
\hline 1556829 & U. californica, Alameda & 20.4 & 29.3 & 34.3 & N/A & N/A & N/A & N/A & N/A & N/A & P. nemorosa \\
\hline $1470622-10$ & Osmanthus fragrans, Sacramento & 16.3 & $\begin{array}{c}34.6 \\
(1 / 20)\end{array}$ & 27.1 & N/A & N/A & N/A & N/A & 31.8 & N/A & P. pini \\
\hline $1309588-15$ & Rhododendron sp., Los Angeles & 19.2 & 23.1 & 23.1 & N/A & N/A & N/A & N/A & 26 & N/A & P. plurivora \\
\hline $1543961-25$ & Rhododendron sp., Orange & 21.1 & 28 & 26.5 & N/A & N/A & N/A & N/A & 30.1 & N/A & P. plurivora \\
\hline $1426752-6$ & Rhododendron sp., San Diego & 21.1 & 24.7 & 24.9 & N/A & N/A & N/A & N/A & 29.4 & 45.9 & P. plurivora \\
\hline $1404765-11$ & Laurus nobilis, Sacramento & 16.5 & 29.9 & 27.8 & N/A & N/A & N/A & N/A & N/A & N/A & $\begin{array}{l}\text { P. sp. aff. } \\
\text { colocasiae } 1^{\mathrm{d}}\end{array}$ \\
\hline $1470622-9$ & Pieris japonica, Sacramento & 14.7 & 22 & 23.4 & N/A & N/A & N/A & N/A & 27.8 & N/A & $\begin{array}{l}\text { P. sp. aff. } \\
\text { colocasiae } 1^{\mathrm{d}}\end{array}$ \\
\hline $1290221-19$ & Unknown, Amador & 19.8 & 24.6 & 26.7 & N/A & N/A & N/A & N/A & N/A & N/A & $\begin{array}{l}\text { P. sp. aff. } \\
\text { colocasiae } 1^{\mathrm{d}}\end{array}$ \\
\hline 1537555 & Sequoia sempervirens, Madera & 22.2 & 27.8 & 27.6 & N/A & N/A & N/A & N/A & N/A & N/A & P. austrocedrae \\
\hline $1556835-2$ & Umbellularia californica, Alameda & 19.3 & 22.7 & 24.9 & N/A & N/A & 31.9 & N/A & N/A & 26.8 & P. pseudosyringae ${ }^{\mathrm{c}}$ \\
\hline $1504925-1$ & U. californica, Monterey & 17.8 & 22.5 & 24.5 & N/A & N/A & N/A & N/A & N/A & 26.6 & P. pseudosyringae \\
\hline $1504925-19$ & U. californica, Monterey & 19.9 & 43.3 & 39.3 & N/A & N/A & N/A & N/A & N/A & 42.1 & P. pseudosyringae \\
\hline $1543964-18$ & Rhododendron sp., Orange & 22.1 & $\begin{array}{l}31.1 \\
(1 / 20)\end{array}$ & 32.9 & N/A & N/A & N/A & N/A & N/A & N/A & P. foliorum ${ }^{\mathrm{c}}$ \\
\hline 1543964-19 & Rhododendron sp., Orange & 21 & 44.7 & 37.2 & N/A & N/A & N/A & N/A & N/A & N/A & P. foliorum \\
\hline $1483418-8$ & Rhododendron sp., Stanislaus & 20.1 & 25.1 & 26.2 & N/A & N/A & N/A & N/A & N/A & N/A & P. foliorum ${ }^{\mathrm{c}}$ \\
\hline $1322287-3$ & Camellia japonica, Santa Clara & 19.5 & 36.9 & 25.8 & N/A & N/A & N/A & N/A & N/A & N/A & P. hibernalis \\
\hline $1543964-25$ & Rhododendron sp., Orange & 20.7 & 39.7 & 25.5 & N/A & N/A & N/A & N/A & N/A & N/A & P. hibernalis \\
\hline $1413767-4$ & Rhododendron sp., Solano & 21.3 & 21.9 & 22 & N/A & N/A & N/A & N/A & N/A & N/A & P. hibernalis \\
\hline 1504971 & Xylosma sp., Marin & 18.4 & 24.5 & 25.1 & N/A & N/A & N/A & N/A & N/A & N/A & P. hibernalis \\
\hline $1309587-25$ & $\begin{array}{l}\text { Schefflera actinophylla, } \\
\text { Los Angeles }\end{array}$ & 16.3 & 22.8 & 21.9 & N/A & N/A & N/A & N/A & N/A & N/A & 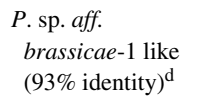 \\
\hline $1309587-26$ & S. actinophylla, Los Angeles & 17.2 & 34.8 & 25.9 & N/A & N/A & N/A & N/A & N/A & N/A & $\begin{array}{l}\text { P. sp. aff. } \\
\text { brassicae-1 like } \\
(93 \% \text { identity) }\end{array}$ \\
\hline $1470362-41$ & Kalmia sp., Sacramento & 20 & 33.9 & 27 & N/A & N/A & 31 & N/A & N/A & N/A & P. tropicalis $^{\mathrm{c}}$ \\
\hline 1556826 & Osmanthus fragrans, Alameda & 15.3 & 21.1 & 22.1 & N/A & N/A & N/A & N/A & 27.6 & N/A & $\begin{array}{l}\text { No homology } \\
\text { in database }\end{array}$ \\
\hline $1518453-1$ & Quercus sp., Merced & 18.6 & 44 & 32.4 & N/A & N/A & N/A & N/A & N/A & N/A & $\begin{array}{l}\text { No homology } \\
\text { in database }\end{array}$ \\
\hline $1364175-13$ & R. californica, Santa Clara & 19 & $\begin{array}{c}31.4 \\
(1 / 20)\end{array}$ & 31.6 & N/A & N/A & N/A & N/A & $\begin{array}{l}38.5 \\
\text { (und) }\end{array}$ & 43.1 & PS \\
\hline $1364175-12$ & Rhamnus californica, Santa Clara & 17.7 & 47.2 & 30.6 & N/A & N/A & N/A & N/A & N/A & N/A & PS \\
\hline 1422489 & Rhododendron sp., Alameda & 16.1 & 19.1 & 21.7 & N/A & N/A & 25.8 & N/A & N/A & N/A & $\begin{array}{l}\text { PS-CDFA + } \\
P \text {. syringae culture }\end{array}$ \\
\hline $1261274-2$ & Tristania sp., Riverside & 19.7 & 46 & 33.1 & N/A & N/A & N/A & N/A & N/A & N/A & PS \\
\hline
\end{tabular}


for an additional 58 described and 12 provisional species (putative probes in Supplementary Table 2), including some species in the $P$. citricola complex (P. citricola, $P$. multivora, and $P$. plurivora). This means that species-specific markers should be able to be developed for $>75 \%$ of the species evaluated in this study and, with the use of locked nucleic acids in the probe, the number species-specific probes may increase.

A number of real-time PCR assays have been developed for detection of Phytophthora spp., most of which have used primarily SYBR Green or TaqMan technology and either the ITS or Ypt1 locus as a target for systematically developing primers or probes (32). The mitochondrial assays described herein offer several advantages over those previously available, perhaps most important of which is the genus-specific detection capability. Although a real-time PCR Phytophthora genus-specific detection capability has been reported for an ITS-based detection assay, background detection of Pythium spp. was noted (26). The use of loci spanning conserved mitochondrial gene-order differences for Phytophthora spp. has eliminated this problem for the two diagnostic markers described in this work. Although there were two species that were not amplified by the atp9-nad9 primer pair, all species were amplified and detected by the trnM-trnM detection assay. The ability to confirm whether a Phytophthora sp. is present with the use of the genus-specific amplicon rather than just test for a particular species will improve diagnostic capabilities and facilitate a better understanding of the involvement of Phytophthora spp. in agricultural and natural ecosystems. With the atp9-nad9 locus, it will be possible to identify unknown species without culturing by sequencing the amplicon and comparing the data with the sequence database representing $>720$ isolates of 114 distinct phylogenetic entities (F. Martin, unpublished data). When more than one species is present, such as with samples recovered by baiting from streams, cloning may be necessary to generate useful sequence data.

TABLE 7. Results of real-time polymerase chain reaction assays for samples collected from Big Sur, California using the Phytophthora genus, P. ramorum and P. kernoviae species-specific and plant-positive control TaqMan assays

\begin{tabular}{|c|c|c|c|c|c|c|c|c|}
\hline \multirow[b]{2}{*}{ Isolate $^{\mathrm{a}}$} & \multirow[b]{2}{*}{ Host, species } & \multirow[b]{2}{*}{ Symptoms } & \multicolumn{2}{|c|}{ P. ramorum } & \multicolumn{4}{|c|}{ Cycle threshold ${ }^{\mathrm{b}}$} \\
\hline & & & Culture PARP-V8 & Dilution & Phy-Genus & P. ramorum & P. kernoviae & Plant \\
\hline BS1 & Tan oak & Leaf spots & - & $1 / 10$ & N/A & N/A & N/A & 23.5 \\
\hline BS 2 & Bay laurel & Leaf spots & + & $1 / 10$ & 30.4 & 29.6 & N/A & 24.5 \\
\hline BS 4 & Maple & Leaf spots & - & $1 / 10$ & N/A & N/A & N/A & 23.3 \\
\hline BS 5 & Bay laurel & Leaf spots & + & $1 / 10$ & 26.8 & 26.7 & N/A & 24.4 \\
\hline BS 6 & Bay laurel & Leaf spots & - & $1 / 10$ & 26.8 & 26.6 & N/A & 22.7 \\
\hline BS 12 & Tan oak & Leaf spots & - & $1 / 10$ & N/A & N/A & N/A & 20.2 \\
\hline BS 13 & Tan oak & Leaf spots & - & $1 / 10$ & N/A & N/A & N/A & 20.5 \\
\hline BS 14 & Maple & No symptoms & - & $1 / 10$ & N/A & N/A & N/A & 22.1 \\
\hline BS 15 & Bay laurel & No symptoms & - & $1 / 10$ & N/A & N/A & N/A & 21.8 \\
\hline BS 16 & Live oak & Few leaf spots & - & $1 / 10$ & N/A & N/A & N/A & 22.9 \\
\hline BS 17 & Bay laurel & Leaf spots & + & $1 / 10$ & 25.3 & 24.6 & N/A & 23.7 \\
\hline P10130 & P. ramorum & $\ldots$ & $0.039000 \mathrm{ng} / \mu \mathrm{l}$ & $10^{-2}$ & 28.1 & 28 & N/A & N/A \\
\hline P10130 & P. ramorum & $\ldots$ & $0.003900 \mathrm{ng} / \mu \mathrm{l}$ & $10^{-3}$ & 36 & 32.3 & N/A & N/A \\
\hline P10130 & P. ramorum & $\ldots$ & $0.000390 \mathrm{ng} / \mu \mathrm{l}$ & $10^{-4}$ & N/A & N/A & N/A & N/A \\
\hline P10130 & P. ramorum & $\ldots$ & $0.000039 \mathrm{ng} / \mu \mathrm{l}$ & $10^{-5}$ & N/A & N/A & N/A & N/A \\
\hline P10130 & P. ramorum & $\ldots$ & $0.000004 \mathrm{ng} / \mu \mathrm{l}$ & $10^{-6}$ & N/A & N/A & N/A & N/A \\
\hline 1571 & P. kernoviae & $\ldots$ & $1 \mathrm{ng} / \mu \mathrm{l}$ & $10^{0}$ & 21.7 & N/A & 23.2 & N/A \\
\hline $\mathrm{H}_{2} \mathrm{O}$ & $\ldots$ & $\ldots$ & $\ldots$ & $\ldots$ & N/A & N/A & N/A & N/A \\
\hline
\end{tabular}

a Location of the samples BS1-BS13 were collected in Pfeiffer Big Sur State Park and BS14 to BS20 in Andrew Molera State Park, Big Sur, CA.

${ }^{\mathrm{b}} \mathrm{N} / \mathrm{A}=$ no amplification.

TABLE 8. Results of real-time polymerase chain reaction assays for root samples collected from Rubus sp. in Oregon using the Phytophthora genus, Phytophthora rubi, and P. fragariae species-specific and plant-positive control TaqMan assays

\begin{tabular}{|c|c|c|c|c|c|}
\hline \multirow[b]{2}{*}{ Samples (dilution) } & \multicolumn{5}{|c|}{ Cycle threshold } \\
\hline & TrnM-PhyG & ATP9-PhyGenus & P. rubi & P. fragariae & Plant assay \\
\hline Raspberry root DNA (dil. 1/50)-r1DNA & 26.9 & 29.8 & 29.6 & N/A & 22.9 \\
\hline Raspberry root DNA (dil. 1/50)-r2DNA & 26.8 & 31.9 & 30.4 & N/A & 22.7 \\
\hline Raspberry root DNA (dil. 1/50)-r4DNA & N/A & N/A & N/A & N/A & 24.3 \\
\hline Raspberry root DNA (dil. 1/50)-r5DNA & 26.9 & 30.9 & 29.6 & N/A & 24.5 \\
\hline Raspberry root DNA (dil. 1/50)-r6DNA & 25.3 & 29.1 & 28.1 & N/A & 24 \\
\hline Raspberry root DNA (dil. 1/50)-DNA number 2 & 28.9 & 34.4 & 30.4 & N/A & 32.6 \\
\hline P. rubi isolate $(1 / 10)$ & $-^{\mathrm{a}}$ & 23.1 & 22.3 & N/A & N/A \\
\hline P. fragariae var. frag. $(1 / 100)$ & - & 25.9 & N/A & 25.5 & N/A \\
\hline $\mathrm{H}_{2} \mathrm{O}$ & N/A & N/A & N/A & N/A & N/A \\
\hline
\end{tabular}

a - Indicates that samples were not tested. 
Like the ITS region, the mitochondrial genome is high copy number, thereby improving the sensitivity of detection. However, unlike the nuclear genome, it is uniparentally inherited; thus, caution should be exercised when using these mitochondrial markers to detect hybrid species because the mitochondrial background of a hybrid isolate may differ depending on which species is the maternal parent $(7,17,29,35)$. Therefore, the assay may not provide an accurate detection of hybrids unless coupled with a nuclear marker assay. A species-specific TaqMan probe was developed for one hybrid species, $P$. alni, and found to be specific for all three subspecies ( $P$. alni subspp. alni, multiformis, and uniformis) even though coxl and nadhl gene sequences were different for $P$. alni subsp. multiformis and $P$. alni subsp. uniformis ( $P$. alni subsp. alni had sequences that grouped with either of these subspecies) (23). Sequence alignment of the Phytophthora genus-specific amplicon for multiple isolates of this species revealed a highly conserved region used for the TaqMan probe annealing site (Supplementary alignment file). The parental lineages of this species has yet to be identified (23); therefore, it is unknown whether these would cause a nonspecific background detection when using the $P$. alni-specific TaqMan probe.

The appropriateness of using this marker system for pathogen quantification has yet to be fully explored. It needs to be experimentally determined whether there are different amounts of mitochondria present depending on the age and condition of the lesion, which would influence the linear relationship between $\mathrm{C}_{\mathrm{t}}$ and level of pathogen colonization. Likewise, if the markers are going to be used for pathogen quantification in the soil, the linear relationship between propagule densities and $\mathrm{C}_{\mathrm{t}}$ must be characterized. An internal control has been developed with the atp9nad9 amplification primers spanning unique internal sequences using the same approach as the internal control for a Verticillium dahliae soil quantification assay (4) to evaluate the suitability of this marker system for soil quantification (G. J. Bilodeau, unpublished data). Although the ITS region has been used as a target for real-time PCR quantification assays, the suitability of this locus for this purpose may also be compromised due to variation in copy number. Although variation in copy number of the rDNA repeat has been observed in several Eumycotan fungi $(4,12,28,36,37,39)$ and there is evidence to suggest this occurs with the related genera Pythium (31) and Phytopythium (44), it has not been experimentally verified for Phytophthora.

The multiplexed assay includes a plant positive control, confirming whether the extracted DNA is amplifiable thereby reducing the chance of false negatives. The $\mathrm{C}_{t}$ of the plant amplification can also give some idea of whether PCR inhibitors are affecting amplification efficiency and reducing the sensitivity of pathogen detection. Due to the high amount of plant relative to pathogen DNA in most samples, if the $\mathrm{C}_{t}$ for plant amplification is higher than expected, a sample dilution or additional DNA purification steps may be necessary to be able to accurately detect whether the pathogen is present.

Isolating a Phytophthora sp. from some environmental samples can be difficult, sometimes requiring multiple attempts at different times of year to obtain a viable isolate. One approach diagnosticians use to evaluate whether a Phytophthora sp. is present is an enzyme-linked immunosorbent assay (ELISA)-based test. This has the advantage of giving reliable results year round, even if the tissue has some decay. However, the ELISA-based tests are not entirely genus specific-they can react with some Pythium spp. (32) - therefore, positive results must be scrutinized carefully. It is always useful and sometimes necessary to know what Phytophthora sp. is causing damage on a particular host even when a live culture is unobtainable. For example, overseas shipment of raspberry mother plants must be tested for $P$. rubi before phytosanitary certification can be obtained for export. If an ELISA test for a Phytophthora sp. is positive for one of these plants, a genusspecific PCR test, such as the ones described in this article, can then be used to determine the species infecting the plant by sequencing the amplicon. A species-specific PCR test can also be used to quickly eliminate $P$. rubi from the list of possibilities. Sequencing is now often less expensive and always faster for preliminary identification of a Phytophthora isolate, especially if it is an unfamiliar species.

The multiplexed diagnostic assays described herein provide a useful assay for detection and identification of Phytophthora spp. in environmental samples. The genus-specific detection capability will significantly increase diagnostic capabilities over current real-time PCR assays by providing a broader view of the involvement of this genus in causing disease. It will also provide a useful tool for evaluating the presence of this genus in natural ecosystems in the absence of disease symptoms (e.g., stream baiting assays) and, with the extensive sequence database of the atp9-nad9 locus available, will facilitate species identification by BLAST analysis without the need for culturing. The screening completed to date indicates genus level specificity when tested against $P$. vexans and a range of plant and Pythium spp.; however, additional validation against other oomycetes would be advisable when conducting ecological studies to ensure specificity. Although species-specific detection capabilities were demonstrated for 13 species and one species complex, in silico analysis suggests that species-specific probes can be developed for $\approx 75 \%$ of the species for which sequence data was collected. Given this level of sequence divergence and the available sequence database for the genus, this locus should be useful for the systematic development of markers for newly described species. The design of the marker system with a single pair of amplification primers and different TaqMan probes for genus- and species-specific detection reduces the potential of affecting amplification efficiency when multiple loci are amplified simultaneously. It also simplifies development of multiplex assays because labeling dyes on the TaqMan probe can be adjusted to provide the detection capabilities needed. The atp9-nad9 and trnM-trnM diagnostic assays both support high-throughput sample processing and will provide sequence data that will be useful for development of other molecular diagnostic assays such as macro (13) or micro arrays (43) for pathogen detection. The sequence alignments used for designing species-specific probes may be found in the supplementary material, and atp9-nad9 sequences for additional isolates may be found on the Phytophthora Database (http://www. phytophthoradb.org) and GenBank.

\section{ACKNOWLEDGMENTS}

This project was supported by Agriculture and Food Research Initiative Competitive Grant number 2007-55605-17835 from the USDA National Institute of Food and Agriculture and the USDA Forest Service, Pacific Southwest Research Station, Sudden Oak Death-Phytophthora ramorum Research Program Fiscal Year 2010 funds. We thank N. Grunwald (USDA Agricultural Research Service [ARS], Corvallis, OR), J. Gigot (Washington State University), and V. Fieland (Oregon State University) for providing DNA for P. rubi assay; all the team from Dr. Martin lab's, USDA-ARS, Salinas, CA; and J. Tomlinson, FERA, UK, for providing $P$. kernoviae- and $P$. ramorum-infected plant DNA for validation of the P. kernoviae assay.

\section{NOTE ADDED IN PROOF}

For additional information about phylogenetic species and sequence analysis of the atp9-nad9 locus, see Fungal Genetics and Biology 66:19-32.

\section{LITERATURE CITED}

1. Afonina, I., Ankoudinova, I., Mills, A., Lokhov, S., Huynh, P., and Mahoney, W. 2007. Primers with 5' flaps improve real-time PCR. Biotechniques 43:770, 772, 774.

2. Arif, M., and Ochoa-Corona, F. M. 2012. Comparative assessment of $5^{\prime}$ 
A/T-rich overhang sequences with optimal and sub-optimal primers to increase PCR yields and sensitivity. Mol. Biotechnol. 55:17-26.

3. Bezuidenhout, C. M., Denman, S., Kirk, S. A., Botha, W. J., Mostert, L., and McLeod, A. 2010. Phytophthora taxa associated with cultivated Agathosma, with emphasis on the $P$. citricola complex and $P$. capensis $\mathrm{sp}$. nov. Persoonia: Mol. Phylogeny Evol. Fungi 25:32-49.

4. Bilodeau, G., Koike, S. T., Uribe, P., and Martin, F. N. 2012. Development of an assay for rapid detection and quantification of Verticillium dahliae in soil. Phytopathology 102:331-343.

5. Bilodeau, G., Pelletier, G., Pelletier, F., Lévesque, C. A., and Hamelin, R. C. 2009. Multiplex real-time polymerase chain reaction (PCR) for detection of Phytophthora ramorum, the causal agent of sudden oak death. Can. J. Plant Pathol. 31:195-210.

6. Bilodeau, G. J., Levesque, C. A., de Cock, A. W. A. M., Duchaine, C., Briere, S., Uribe, P., Martin, F. N., and Hamelin, R. C. 2007. Molecular detection of Phytophthora ramorum by real-time polymerase chain reaction using TaqMan, SYBR Green, and molecular beacons. Phytopathology 97:632-642.

7. Blair, J. E., Coffey, M. D., and Martin, F. N. 2012. Species tree estimation for the late blight pathogen, Phytophthora infestans, and close relatives. PLoS One 7:e37003. Online publication. doi:37010.31371/journal/ pone. 0037003

8. Blair, J. E., Coffey, M. D., Park, S. Y., Geiser, D. M., and Kang, S. C. 2008. A multi-locus phylogeny for Phytophthora utilizing markers derived from complete genome sequences. Fungal Genet. Biol. 45:266-277.

9. Brasier, C. M. 2007. Phytophthora biodiversity: how many Phytophthora species are there? Pages 101-115 in: U.S. Dep. Agric. For. Serv. Pac. Southwest Res. Stn. E. M. Goheen and S. J. Frankel, eds. Proceedings of the Fourth Union of Forest Research Organization (IUFRO) Working Party 507.02.09. Gen. Tech. Rep. PSW-GTR-221.

10. Brasier, C. M., Beales, P. A., Kirk, S. A., Denman, S., and Rose, J. 2005. Phytophthora kernoviae sp. nov., an invasive pathogen causing bleeding stem lesions on forest trees and foliar necrosis of ornamentals in the UK. Mycol. Res. 109:853-859.

11. Brasier, C. M., Cooke, D. E. L., and Duncan, J. M. 1999. Origin of a new Phytophthora pathogen through interspecific hybridization. Proc. Natl. Acad. Sci. USA 96:5878-5883.

12. Butler, D. K., and Metzenberg, R. L. 1989. Premeiotic change of nucleolous organizer size in Neurospora. Genetics 122:783-791.

13. Chen, W., Djama, Z. R., Coffey, M. D., Martin, F. N., Bilodeau, G. J., Radmer, L., Denton, G., and Lévesque, C. A. 2012. Membrane-based oligonucleotide array developed from multiple markers for the detection of many Phytophthora species. Phytopathology 103:43-54.

14. Dorak, M. T. 2006. Real-Time PCR. E. Owen and M. T. Dorak, eds. Taylor \& Francis, Oxford.

15. Garbelotto, M., Davidson, J. M., Ivors, K., Maloney, P. E., Huberli, D., Koike, S. T., and D. M. Rizzo 2003. Non-oak native plants are main hosts for sudden oak death pathogen in California. Calif. Agric. 57:18-23.

16. Garbelotto, M., Rizzo, D. M., Hayden, K., Meija-Chang, M., Davidson, J. M., and Tjosvold, S. 2002. Phytophthora ramorum and sudden oak death in California: III. Preliminary studies in pathogen genetics. U. S. Dep. Agric. For. Serv. Gen. Tech. Rep. PSW-GTR-184:765-774.

17. Goss, E. M., Cardenas, M. C., Myers, K., Forbes, G. A., Fry, W. E., Restrepo, S., and Grunwald, N. J. 2011. The plant pathogen Phytophthora andina emerged via hybridization of an unknown Phytophthora species and the Irish potato famine pathogen, P. infestans. PLoS One 6:e24543.

18. Goss, E. M., Larsen, M., Chastagner, G. A., Givens, D. R., Grünwald, N. J., and Howlett, B. J. 2009. Population genetic analysis infers migration pathways of Phytophthora ramorum in US nurseries. PLoS Pathog. 5:e1000583.

19. Hall, T. A. 1999. BioEdit: a user-friendly biological sequence alignment editor and analysis program for Windows 95/98/NT. Nucleic Acids Symp. Ser. 41:95-98.

20. Heid, C., Stevens, J., Livak, K., and Williams, P. 1996. Real-time quantitative PCR. Genome Res. 6:986-994.

21. Hong, C., Gallegly, M. E., Richardson, P. A., and Kong, P. 2011. Phytophthora pini Leonian resurrected to distinct species status. Mycologia 103:351-360

22. Hong, C. X., Gallegly, M. E., Browne, G. T., Bhat, R. G., Richardson, P. A., and Kong, P. 2009. The avocado subgroup of Phytophthora citricola constitutes a distinct species, Phytophthora mengei sp. nov. Mycologia 101:833-840

23. Ioos, R., Andrieux, A., Marcais, B., and Frey, P. 2006. Genetic characterization of the natural hybrid species Phytophthora alni as inferred from nuclear and mitochondrial DNA analyses. Fungal Genet. Biol. 43:511529.

24. Jeffers, S. N., and Martin, S. B. 1986. Comparison of two media selective for Phytophthora and Pythium species. Plant Dis. 70:1038-1043.
25. Jung, T., and Burgess, T. I. 2009. Re-evaluation of Phytophthora citricola isolates from multiple woody hosts in Europe and North America reveals a new species, Phytophthora plurivora sp. nov. Persoonia 22:95-110.

26. Kox, L. F. F., van Brouwershaven, I. R., van de Vossenberg, B., van den Beld, H. E., Bonants, P. J. M., and de Gruyter, J. 2007. Diagnostic values and utility of immunological, morphological, and molecular methods for in planta detection of Phytophthora ramorum. Phytopathology 97:11191129.

27. Kroon, L. P. N. M., Brouwer, H., de Cock, A. W. A. M., and Govers, F. 2012. The genus Phytophthora anno 2012. Phytopathology 102:348-364.

28. Maleszka, R., and Clark-Walker, G. D. 1993. Yeasts have a four-fold variation in ribosomal DNA copy number. Yeast 9:53-58.

29. Man-in-'t-Veld, W. A., Cock, A. W. A. M., and Summerbell, R. C. 2007. Natural hybrids of resident and introduced Phytophthora species proliferating on multiple new hosts. Eur. J. Plant Pathol. 117:25-33.

30. Martin, F. N. 2000. Phylogenetic relationships among some Pythium species inferred from sequence analysis of the mitochondrially encoded cytochrome oxidase II gene. Mycologia 92:711-727.

31. Martin, F. N. 2009. Pythium genetics. Pages 213-223 in: Oomycete Genetics and Genomics. K. Lamour and S. Kamoun, eds. John Wiley and Sons, Hoboken, NJ.

32. Martin, F. N., Abad, Z. G., Balci, Y., and Ivors, K. 2012. Identification and detection of Phytophthora: reviewing our progress, identifying our needs. Plant Dis. 96:1080-1103.

33. Martin, F. N., Bensasson, D., Tyler, B. M., and Boore, J. L. 2007. Mitochondrial genome sequences and comparative genomics of Phytophthora ramorum and P. sojae. Curr. Genet. 51:285-296.

34. Martin, F. N., Tooley, P. W., and Blomquist, C. 2004. Molecular detection of Phytophthora ramorum, the causal agent of sudden oak death in California, and two additional species commonly recovered from diseased plant material. Phytopathology 94:621-631.

35. Nirenberg, H. I., Gerlach, W. F., and Graefenhan, T. 2009. Phytophthora $\times$ pelgrandis, a new natural hybrid pathogenic to Pelargonium grandiflorum hort. Mycologia 101:220-231.

36. Pasero, P., and Marilly, M. 1993. Size variation rDNA clusters in the yeasts Saccharomyces cerevisiae and Saccharomyces pombe. Mol. Gen. Genet. 236:448-452.

37. Pukkila, P. J., and Skrzynia, C. 1993. Frequent changes in the number of reiterated ribosomal RNA genes throughout the lifecycle of the basidiomycete Coprinus cinereus. Genetics 133:203-211.

38. Rizzo, D. M., and Garbelotto, M. 2003. Sudden oak death: endangering California and Oregon forest ecosystems. Front. Ecol. Environ. 1:197204.

39. Rustchenko-Bulgac, E. P., Curran, T., and Sherman, F. 1993. Variation in the number of ribosomal DNA subunits in morphological mutants and normal strains of Candida albicans and in normal strains of Saccharomyces cerevisiae. J. Bacteriol. 175:7189-7199.

40. Schena, L., Duncan, J. M., and Cooke, D. E. L. 2008. Development and application of a PCR-based 'molecular tool box' for the identification of Phytophthora species damaging forests and natural ecosystems Plant Pathol. 57:64-75.

41. Schena, L., Hughes, K. J. D., and Cooke, D. E. L. 2006. Detection and quantification of Phytophthora ramorum, $P$. kernoviae, $P$. citricola and $P$. quercina in symptomatic leaves by multiplex real-time PCR. Mol. Plant Pathol. 7:365-379.

42. Scott, P. M., Burgess, T. I., Barber, P. A., Shearer, B. L., Stukely, M. J. C., Hardy, G. E. S. J., and Jung, T. 2009. Phytophthora multivora sp. nov., a new species recovered from declining Eucalyptus, Banksia, Agonis and other plant species in Western Australia. Persoonia 22:1-13

43. Sikora, K., Verstappen, E., Mendes, O., Schoen, C., Ristaino, J., and Bonants, P. 2012. A universal microarray detection method for identification of multiple Phytophthora spp. using padlock probes. Phytopathology 102:635-645

44. Spies, C. F. J., Mazzola, M., and McLeod, A. 2011. Characterisation and detection of Pythium and Phytophthora species associated with grapevines in South Africa. Eur. J. Plant Pathol. 131:103-119.

45. Tooley, P. W., Martin, F. N., Carras, M. M., and Frederick, R. D. 2006. Real-time fluorescent polymerase chain reaction detection of Phytophthora ramorum and Phytophthora pseudosyringae using mitochondrial gene regions. Phytopathology 96:336-345.

46. USDA-APHIS 2004. Sudden oak death: Phytophthora ramorum. In: Pest Detection and Management Programs. L. Levy and V. Mavrodieva, eds. USDA Animal Plant Health Inspection Service, USA.

47. White, T. J., Bruns, T. D., Lee, S. B., and Taylor, J. W. 1990. Amplification and direct sequencing of fungal ribosomal RNA Genes for phylogenetics. Pages 315-322 in: PCR Protocols: A Guide to Methods and Applications-A Laboratory Manual. N. Innis, D. Gelfand, J. Sninsky, and T. White, eds. Academic Press, New York. 